\title{
Therapie der Multiplen Sklerose
}

\author{
4.1 Stufentherapie -56 \\ Miriam Kip, Heinz Wiendl
}

4.1.1 Therapieziele und Übersicht empfohlener Therapieansätze - 57

4.1.2 Prinzipien der Stufentherapie -59

4.1.3 Versorgungssituation -61

4.2 Symptomatische Therapie und Rehabilitation körperlicher und kognitiver Symptome $\quad-75$

Miriam Kip, Anne Talaschus, Iris-Katharina Penner

4.2.1 Therapieziele und Übersicht empfohlener Therapieansätze - 76

4.2.2 Prinzipien der Rehabilitation -76

4.2.3 Versorgungssituation -82

Literatur $\quad-89$ 


\subsection{Stufentherapie}

Miriam Kip, Heinz Wiendl

\section{Zusammenfassung}

Die Stufentherapie beinhaltet die (dauerhafte) Arzneimitteltherapie zur Reduktion der Anzahl der Schübe (Schubprophylaxe, verlaufsmodifizierende Therapie) und die Akut-Therapie des Schubes. Dabei wird idealerweise ein Zustand frei von Krankheitsaktivität angestrebt (no evidence of disease activity, NEDA). Sie richtet sich nach der Verlaufsform der Erkrankung und hat das Ziel, die Progression der Erkrankung zu verhindern. Die Primärversorgung der Patienten mit Multipler Sklerose erfolgt durch Neurologen, und in den meisten Fällen wird die verlaufsmodifizierende Therapie ambulant durchgeführt. Seit 2011 hat mit dem Hinzukommen neuer therapeutischer Möglichkeiten bei der Behandlung der schubförmig verlaufenden MS (RRMS) der indikationsbezogene Arzneimittelverbrauch unter gesetzlich Versicherten in Deutschland jährlich zugenommen. Die Versorgungssituation in der Therapie von Patienten mit Erstdiagnose einer MS ist dennoch unzureichend. Studien, basierend auf Versichertendaten der gesetzlichen Krankenkasse, deuten darauf hin, dass zu wenig Patienten mit Erstdiagnose einer MS zeitnah auch eine Therapie erhalten. Lediglich jeder zweite Patient mit dokumentierter Erstmanifestation einer MS in 2009 nahm im selben Jahr eine verlaufsmodifizierende Therapie in Anspruch. In Abhängigkeit von der regionalen Facharztdichte suchten zwischen $37 \%$ und $64 \%$ der Patienten innerhalb der ersten sechs Wochen nach Erstdiagnose überhaupt einen Neurologen für die Weiterbehandlung auf. Der frühe Therapiebeginn ist aber für die erfolgreiche Behandlung wichtig. Es ist anzustreben, Patienten nach erstmaligem demyelinisierendem Ereignis (klinischisoliertes Syndrom) medikamentös zu behandeln, da dies beispielsweise eine Konversion in eine RRMS verzögert bzw. die Wahrscheinlichkeit längerfristiger Behinderung reduziert. In der Behandlung der RRMS geben Studien Hinweise auf eine Unter- und mögliche Fehlversorgung. Gut $40 \%$ der Patienten der gesetzlichen Krankenversicherung mit dokumentierter RRMS nahmen 2009 überhaupt keine verlaufsmodifizierende Therapie in Anspruch. Die Behandlung der RRMS soll sich entsprechend den 2014 geänderten
Handlungsempfehlungen der Deutschen Gesellschaft für Neurologie an der Krankheitsaktivität orientieren. Rund $15 \%$ der Patienten mit MS innerhalb der gesetzlichen Krankenversicherung werden jährlich akut-stationär behandelt. Gut zwei Drittel der wegen einer MS stationär behandelten Patienten nahmen sechs Monate vor dem Krankhausaufenthalt keine verlaufsmodifizierende Therapie in Anspruch. Bei $27 \%$ dieser Patienten wurde nach dem Krankenhausaufenthalt eine verlaufsmodifizierende Therapie initiiert. Bei gut einem Viertel der mit Medikamenten bei niedriger Krankheitsaktivität behandelten Patienten mit RRMS, die klinisch unauffällig waren, waren Zeichen erhöhter Krankheitsaktivität im MRT messbar. Entsprechend den aktuell geänderten Empfehlungen einer an die Krankheitsaktivität angepassten Therapie können diese Patienten von einer Therapie mit Medikamenten für eine (hoch-) aktive Verlaufsform profitieren. Die Adhärenz hinsichtlich der verlaufsmodifizierenden Therapie ist niedrig. Zwischen 2002 und 2006 betrug sie unter gesetzlich Versicherten zwischen 30 und $40 \%$. Nur ein Drittel der Patienten führte die Therapie für einen Zeitraum von zwei Jahren auch kontinuierlich durch. Als Hemmnisse der Adhärenz werden vor allen Dingen Nebenwirkungen, eine nachgewiesene oder angenommen fehlende Wirksamkeit der Therapie, aber auch Begleiterkrankungen wie die Fatigue oder Depressionen genannt. Es gibt Hinweise, dass die Akutbehandlung des Schubes zu häufig stationär erfolgt. Eine Auswertung des Datensatzes der Deutschen Multiple Sklerose Gesellschaft ergab, dass lediglich gut ein Drittel der Schubtherapien ambulant durchgeführt wird, wobei die Wahrscheinlichkeit einer ambulanten Therapie mit der Krankheitsdauer zunahm. Aus Sicht von Patienten und Ärzten ist die fehlende Verfügbarkeit wirksamer Medikamente für die Behandlung progredienter Verlaufsformen ein dringender Versorgungsbedarf. Denn mit Zunahme der Progredienz der Erkrankung nehmen die therapeutischen Möglichkeiten innerhalb der verlaufsmodifizierenden Therapie ab. Für die Therapie der primär-progredienten MS sind in Deutschland derzeit formal keine Medikamente zugelassen.

Gegenwärtig sind keine kurativen Therapien für die Multiple Sklerose (MS) bekannt. Für die meisten Betroffenen existieren aber wirksame Therapie- 
ansätze, um die Krankheitsaktivität zu reduzieren, den Krankheitsverlauf günstig zu beeinflussen und die Symptome der Erkrankung zu behandeln. Die Stufentherapie beschreibt Behandlungspfade getrennt nach Verlaufsform der MS (schubförmige, sekundär und primär progrediente MS) und in Abhängigkeit der Krankheitsaktivität. Bei der schubförmigen Verlaufsform (relapsing remitting MS, RRMS) setzt sie sich aus der verlaufsmodifizierenden Therapie für die Schubprophylaxe und der Therapie des akuten Schubs zusammen. Sie umfasst medikamentöse Therapieansätze, die spezifisch und unspezifisch auf das Immunsystem wirken.

Das folgende Kapitel beschreibt die Versorgungssituation hinsichtlich der Inanspruchnahme der Stufentherapie von Patienten mit MS. Abschnitt eins erläutert die Therapieziele und gibt eine Übersicht über die derzeit empfohlenen Arzneimittel für die verlaufsmodifizierende Therapie. Abschnitt zwei beschreibt die Prinzipien der Stufentherapie unter Berücksichtigung aktueller Entwicklungen im Bereich der Verlaufsformen und der Krankheitsaktivität. Abschnitt drei beschreibt die Inanspruchnahme der Stufentherapie unter Berücksichtigung von Aspekten der Adhärenz und der besonderen Behandlungssituation bei einem Kinderwunsch und einer Schwangerschaft. Dabei werden aktuelle Behandlungsempfehlungen der Versorgungsrealität gegenübergestellt.

\subsubsection{Therapieziele und Übersicht empfohlener Therapieansätze}

Die Stufentherapie der RRMS gliedert sich in die verlaufsmodifizierende Therapie und die Therapie des Schubs. Sie hat das Ziel, die Krankheitsaktivität und Schubhäufigkeit zu reduzieren und damit die Wahrscheinlichkeit langfristiger Krankheitssymptome zu senken. Die Therapie strebt an, irreversible neurologische Schäden und körperliche Behinderungen sowie neurokognitive und psychische Veränderungen unter Berücksichtigung der Lebensqualität und Patientenautonomie zu verhindern (DGN 2014, Gold et al. 2012). Dabei ist neben der Kontrolle der klinischen Krankheitsaktivität auch die Kontrolle der paraklinischen Aktivität ein er- klärtes Ziel (no evidence of disease activity NEDA).

Für die Darstellung der Arzneimittel zur Therapie der MS nehmen wir Bezug auf die aktuelle S2e-Leitlinie zur Diagnose und Therapie der Multiplen Sklerose der Deutschen Gesellschaft für Neurologie (DGN) mit Stand Januar 2012. In der Online-Ausgabe der Leitlinie wurden im Jahr 2014 einige Änderungen zur Stufentherapie und seit dem Jahr 2012 neu zugelassene Arzneimittel für die Therapie der MS ergänzend aufgenommen (DGN 2014) (•Tab. 4.1).

Die neu für die Behandlung der MS zugelassenen Medikamente erweitern das Spektrum therapeutischer Möglichkeiten, insbesondere bei Versagen der herkömmlichen Medikamente und (hoch) aktiven Verläufen der RRMS. Die meisten der bislang verfügbaren Medikamente mussten subkutan (s.c.), intramuskulär (i.m.) oder intravenös (i.v.) verabreicht werden. Neue oral verfügbare Medikamente zielen zusätzlich auf eine verbesserte Therapieadhärenz (Wilson et al. 2015).

Aufgrund der multifaktoriellen und in Teilen ungeklärten Pathogenese der Erkrankung lassen sich individuelle Krankheitsverläufe nicht sicher voraussagen. Faktoren wie das gleichzeitige Auftreten mehrerer Symptome, die Betonung motorischer Funktionseinschränkungen, lang andauernde Schübe wie auch eine hohes Ausmaß messbarer Krankheitsaktivität in bildgebenden und elektrophysiologischen Verfahren, werden mit einer schlechteren Prognose in Zusammenhang gebracht. Letztendlich sind aber aktuell keine Risikofaktoren identifiziert, mit denen sich verlässlich das individuelle Schubrisiko und der weitere Verlauf und damit das Morbiditäts- und Mortalitätsrisiko von Patienten mit MS vorhersagen ließe (DGN 2014). Die neuen verfügbaren medikamentösen Behandlungsansätze sind mit der therapeutischen Unsicherheit verbunden, ob langfristig die Progression körperlicher und neurokognitiver Funktionseinschränkungen günstig beeinflusst wird (Filippini et al. 2013, Vogel 2015). Unumstritten ist aber, dass eine frühe und an die Krankheitsaktivität angepasste Therapie sich günstig auf den weiteren Krankheitsverlauf auswirkt (Wiendl und Meuth 2015). 
- Tab. 4.1 Übersicht* der derzeit empfohlenen Arzneimittel für die verlaufsmodifizierende Therapie bei MS (Immuntherapeutika, inklusive Glukokortikoide für die Therapie des Schubs)

\begin{tabular}{|c|c|}
\hline Arzneimittel & Beschreibung \\
\hline Alemtuzumab & $\begin{array}{l}\text { Monoklonaler Antikörper mit Bindung an Zelloberfläche von Lymphozyten. } \\
\text { Darreichung parenteral (intravenös). Seit } 2013 \text { für MS zugelassen }\end{array}$ \\
\hline Azathioprin & $\begin{array}{l}\text { Hemmt Lymphozyten-Differenzierung und -Aktivierung. Darreichung oral. } \\
\text { Markteinführung 1967, seit } 2000 \text { für MS zugelassen }\end{array}$ \\
\hline Cyclophosphamid & $\begin{array}{l}\text { Alkylierendes Zytostatikum (N-Lost-Derivat). Verhindert Vermehrung der Körper- } \\
\text { zellen. Darreichung parenteral (intravenös). Markteinführung } 1958 \text { (keine Zulas- } \\
\text { sung für MS) }\end{array}$ \\
\hline Dimethylfumarat & Fumarsäureesther. Darreichung oral. Markteinführung 2014 \\
\hline Fingolimod & Hemmt die Lymphozytenmigration. Darreichung oral. Markeinführung 2011 \\
\hline Glatirameracetat & $\begin{array}{l}\text { Synthetisches Polypeptid, Acetatsalz aus Aminosäuren. Darreichung parenteral } \\
\text { (subkutan). Markteinführung } 2001\end{array}$ \\
\hline Interferon beta-1a Präparate & $\begin{array}{l}\text { Natürlich vorkommendes Protein. Darreichung parenteral (subkutan oder intra- } \\
\text { muskulär). Markteinführung } 1997\end{array}$ \\
\hline Interferon beta-1b Präparate & $\begin{array}{l}\text { Natürlich vorkommendes Protein. Darreichung parenteral (subkutan). Marktein- } \\
\text { führung } 1996\end{array}$ \\
\hline Intravenöse Immunglobuline & $\begin{array}{l}\text { Blutplasmaderivat. Darreichung parenteral (intravenös). Markteinführung } 1962 \\
\text { (Keine Zulassung für MS, Off-Label-Use in der Regel nicht anerkannt) }\end{array}$ \\
\hline Methotrexat & $\begin{array}{l}\text { Antimetabolit, unspezifisches Zytostatikum. Darreichung parenteral (intravenös). } \\
\text { Markteinführung } 1958 \text { (keine Zulassung für MS) }\end{array}$ \\
\hline Methylprednisolon & $\begin{array}{l}\text { Glukokortikoid, Steroidhormon. Darreichung oral oder parenteral (intravenös). } \\
\text { Markteinführung } 1959\end{array}$ \\
\hline Mitoxantron & $\begin{array}{l}\text { Synthetisches Anthrazyklin. Darreichung parenteral (intravenös). Markteinführung } \\
\text { 1985, für MS seit } 2001 \text { zugelassen }\end{array}$ \\
\hline Natalizumab & $\begin{array}{l}\text { Monoklonaler Antikörper. Darreichung parenteral (intravenös). Markteinführung } \\
2006\end{array}$ \\
\hline PEG-Interferon beta-1a & $\begin{array}{l}\text { Pegylierte Form des Interferon beta-1a. Darreichung parenteral (subkutan). } \\
\text { Markteinführung } 2014\end{array}$ \\
\hline Teriflunomid & $\begin{array}{l}\text { Reversible Hemmung des Zellmetabolismus. Darreichung oral. Markteinführung } \\
2013\end{array}$ \\
\hline \multicolumn{2}{|c|}{ 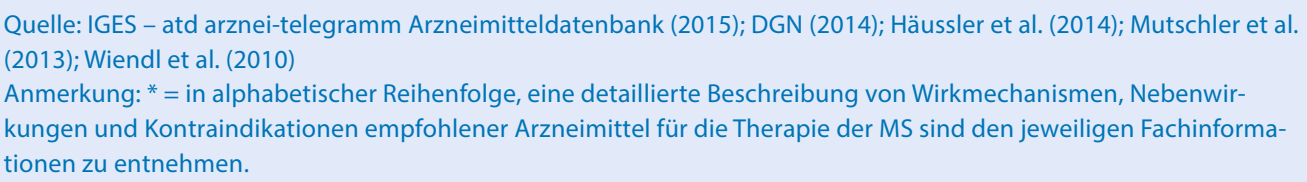 } \\
\hline
\end{tabular}




\begin{tabular}{|c|c|c|c|c|c|c|c|}
\hline \multirow{4}{*}{ 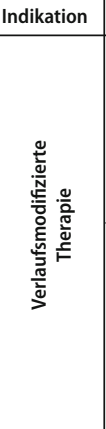 } & \multicolumn{2}{|r|}{$\mathrm{KIS}^{1}$} & \multicolumn{3}{|c|}{ RRMS $^{1}$} & \multicolumn{2}{|c|}{ SPMS $^{1}$} \\
\hline & \multirow[b]{2}{*}{ 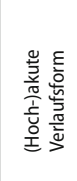 } & & 1. Wahl & 2. Wahl & 3. Wahl & \multirow[t]{2}{*}{$\begin{array}{c}\text { mit } \\
\text { aufgesetzten Schüben }\end{array}$} & \multirow[t]{2}{*}{$\begin{array}{c}\text { ohne } \\
\text { aufgesetzten Schüben }\end{array}$} \\
\hline & & & $\begin{array}{l}\text { - Alemtuzumab } \\
\text { - Fingolimod } \\
\text { - Natalizumab }\end{array}$ & $\begin{array}{l}\text { - Mitoxantron } \\
\text { (-Cyclophosphamid })^{4}\end{array}$ & $\begin{array}{l}\text { - Experimentelle } \\
\text { Verfahren }\end{array}$ & & \\
\hline & 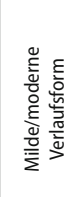 & $\begin{array}{l}\text { - Glatirameracetat } \\
\text { - Interferon- } \beta \text { 1a im } \\
\text { - Interferon- } \beta \text { 1 a sc } \\
\text { - Interferon- } \beta \text { 1b sc }\end{array}$ & $\begin{array}{l}\text { - Dimethylfumarat } \\
\text { - Glatirameracetat } \\
\text { - Interferon- } \beta \text { 1 a im } \\
\text { - Interferon- } \beta \text { 1 a sc } \\
\text { - Interferon- } \beta \text { 1 b sc } \\
\text { - PEG-IFN- } \beta \text { 1a sc } \\
\text { - Teriflunomid } \\
\text { (-Azathioprion) }^{2} \\
(\text {-IVlg) }\end{array}$ & & & $\begin{array}{l}\text { - Interferon- } \beta \text { 1a sc } \\
\text { - Interferon- } \beta \text { 1b sc } \\
\text { - Mitoxantron } \\
\text { (-Cyclophosphamid) }{ }^{4}\end{array}$ & $\begin{array}{l}\text { - Mitoxantron } \\
\text { (-Cyclophosphamid) }\end{array}$ \\
\hline \multirow{2}{*}{ 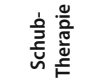 } & \multicolumn{3}{|l|}{ 2. Wahl } & \multicolumn{2}{|l|}{ - Plasmaseparation } & & \\
\hline & \multicolumn{3}{|l|}{ 1. Wahl } & \multicolumn{2}{|c|}{ - Methylprednisolonpuls } & & \\
\hline
\end{tabular}

- Abb. 4.1 Stufentherapie der MS; Anmerkung: $K I S=$ Klinisch isoliertes Syndrom, RRMS = relapsing-remitting MS, SPMS = sekundär progrediente MS. Bei Versagen einer verlaufsmodifizierenden Therapie bei milder/moderater Verlaufsform einer MS werden diese Patienten wie bei einer aktiven MS behandelt. 'Substanzen in alphabetischer Reihenfolge; die hier gewählte Darstellung impliziert KEINE Überlegenheit einer Substanz gegenüber einer anderen innerhalb einer Indikationsgruppe (dargestellt innerhalb eines Kastens). ${ }^{2}$ zugelassen, wenn Interferon beta nicht möglich oder unter Azathioprin-Therapie stabiler Verlauf erreicht. ${ }^{3}$ Einsatz nur postpartal im Einzelfall gerechtfertigt, insbesondere vor dem Hintergrund fehlender Behandlungs-alternativen. ${ }^{4}$ zugelassen für bedrohlich verlaufende Autoimmunkrankheiten, somit lediglich nur für fulminante Fälle als Ausweichtherapie vorzusehen, idealerweise nur an ausgewiesenen MS-Zentren.

Quelle: IGES - Mit freundlicher Genehmigung des Krankheitsbezogenes Kompetenznetz Multiple Sklerose (KKNMS)

DGN (2014)

\subsubsection{Prinzipien der Stufentherapie}

Die Stufentherapie gliedert sich in die verlaufsmodifizierende Therapie (Schubprophylaxe) und die Therapie des akuten Schubes bei schubförmig verlaufender Erkrankung (• Abb. 4.1).

Das therapeutische Vorgehen richtet sich dabei nach der Verlaufsform der MS und der Krankheitsaktivität. Hieraus ergeben sich innerhalb der Stufentherapie unterschiedliche Behandlungspfade (DGN 2014; Vogel 2015).

In den aktuellen Empfehlungen wurden neu zugelassene Medikamente (Alemtuzumab, Dimethylfumarat, Fingolimod, Teriflunomid) aufgenommen und das Prinzip der Basis- und Eskalationstherapie zugunsten einer Orientierung an der Krankheitsaktivität verlassen (DGN 2014).

Die Entscheidung über die verlaufsmodifizierende Therapie wird von Arzt und Patient zu Beginn der Therapie gefällt und muss im Verlauf der Erkrankung vor dem Hintergrund von Nutzen (Wirksamkeit) und Risiken (Nebenwirkungen) immer wieder neu überprüft werden (Butler et al. 2015; DGN 2014).
Die Behandlung des akuten Schubs wird für drei bis fünf Tage mit Kortison und ggf. für weitere fünf Tage bei Nichtansprechen durchgeführt (KortisonStoßtherapie). Als Mittel der zweiten Wahl steht die Plasmaseparation oder alternativ die Immunapharese zur Verfügung (DGN 2014).

\section{Einteilung der Multiplen Sklerose nach Verlaufsformen}

Die seit dem Jahr 1996 gültige Klassifikation der MS erfuhr in den Jahren 2010 und 2014 eine Neuordnung mit den Zielen, eine bessere Vergleichbarkeit der Patienten zu erreichen, die Kommunikation über die Krankheit zu verbessern, Prognosen zu treffen und erfolgreichere Interventionsstudien und Behandlungsentscheidungen $\mathrm{zu}$ etablieren. Zur schubförmig verlaufenden MS (relapsing remitting MS, RRMS) kam das klinisch isolierte Syndrom (KIS) hinzu und die progressive Form der MS wird in primär und sekundär (PPMS, SPMS) unterteilt (Lublin et al. 2014).

Das KIS beschreibt das erstmalige Auftreten eines klinischen Schubs und nachweisbarer disseminierter entzündlicher Demyelinisierung. Das KIS 
kann sich zu einer MS entwickeln, wenn eine zeitliche Dissemination auftritt, d.h. wenn es zu neuen klinischen Symptomen oder neuen Läsionen in der Bildgebung kommt (Lublin et al. 2014). Eine Längsstudie zeigte, dass $82 \%$ der Patienten mit KIS und nachweisbaren MS-typischen Läsionen im MRT und $21 \%$ ohne pathologische MRT-Veränderungen im Verlauf von 20 Jahren eine MS entwickelten (Fisniku et al. 2008a). Die frühzeitige Behandlung, d.h. die Behandlung von Patienten mit KIS, senkt das Risiko eines erneuten klinischen Schubes oder neuer Läsionen im MRT und die Wahrscheinlichkeit einer Konversion in eine MS wird reduziert (Lublin et al. 2014).

Der schubförmige Verlauf der MS (relapsingremitting MS, RRMS) ist die häufigste Verlaufsform. Bei über $80 \%$ der Patienten wird zum Zeitpunkt der Diagnosestellung eine RRMS diagnostiziert. Im Verlauf kommt es wiederkehrend zu einer akuten klinischen Verschlechterung, zu neuen Läsionen in der MRT-Bildgebung und/oder Entmarkungen (Demyelinisierungen), die in elektrophysiologischen Untersuchungen nachgewiesen werden ( $\checkmark$ Kap. 3). Die neurologischen Funktionseinschränkungen bilden sich nach Abklingen des Schubs ganz (komplette Remission) oder teilweise wieder zurück. Die zwischen den Schüben liegenden beschwerdefreien Intervalle sind von unterschiedlicher Dauer (Lublin et al. 2014). Im natürlichen, unbehandelten Verlauf geht nach schätzungsweise 15 bis 29 Jahren nach Krankheitsbeginn bei über der Hälfte der Patienten die Erkrankung in einen progredienten Verlauf über (SPMS) (Hurwitz 2011; Weinshenker et al. 1989).

Die SPMS beschreibt eine kontinuierliche, schleichende Verschlechterung. Der SPMS geht immer eine RRMS voraus. Der Übergang zwischen den beiden Verlaufsformen ist fließend, und ein exakter Zeitpunkt kann weder klinisch noch anhand bestimmter biochemischer Marker oder in der Bildgebung bestimmt werden. Die SPMS kann von aufgesetzten Schüben begleitet sein, die meist mit der Krankheitsdauer in der Häufigkeit abnehmen (Lublin et al. 2014).

Bei Vorliegen einer PPMS liegt eine kontinuierliche klinische Verschlechterung ohne vorangegangene Schübe vor. Nur in wenigen Ausnahmefällen werden Schübe beobachtet. Pathologische Untersu- chungen und die MRT-Bildgebung zeigen bei dieser Verlaufsform weniger Entzündungsaktivität als bei der schubförmigen Verlaufsform. Aktuell besteht keine Empfehlung für eine verlaufsmodifizierende Therapie bei der PPMS, da derzeit hierfür keine zugelassenen Therapieoptionen existieren (DGN 2014).

Das radiologisch isolierte Syndrom (RIS) beschreibt eine morphologische Veränderung im MRT ohne klinisches Korrelat, die MS-typischen Symptome entsprechen. Patienten mit einem RIS bedürfen vom Grundsatz noch keiner Therapie, sollten jedoch engmaschig hinsichtlich klinischer und paraklinischer Krankheitsaktivität kontrolliert werden (DGN 2014, Gold et al. 2012).

\section{Krankheitsaktivität}

Die Krankheitsaktivität soll im Verlauf der Erkrankung (mindestens) einmal jährlich anhand klinischer und paraklinischer (im MRT messbarer) Parameter bestimmt werden. Zu den klinischen Kriterien zählen die Schubrate, -dauer, und -intensität, Grad funktioneller Einschränkungen bzw. körperlicher Beeinträchtigungen und Schwere der Symptome. Im MRT werden definierte Kriterien wie Gadolinium (Gd)-aufnehmende Läsionen, neu auftretende oder vergrößerte T2-Läsionen zur Beurteilung der Krankheitsaktivität herangezogen ( $\triangleright$ Kap. 3.4) (Gold et al. 2012; Lublin et al. 2014).

Die Progression der Erkrankung soll anamnestisch, durch standardisierte Erhebungsinstrumente zur Quantifizierung funktioneller Beeinträchtigungen (EDSS, MSFC, - Kap. 3.2) erhoben werden. Paraklinische Zeichen irreversibler neuronaler Schädigungen (axonale Degeneration, Hirnatrophie) in elektrophysiologischen Verfahren und im MRT korrelieren dabei gut mit dem Grad körperlicher Beeinträchtigungen (Fisniku et al. 2008a,b).

Vor dem Hintergrund eines differenzierten Abbildes von Krankheitsaktivität und des therapeutischen Zieles eines Zustandes ohne Krankheitsaktivität (NEDA), schlagen Stangel et al. in einer 2015 erschienen Publikation eine erweiterte Bewertung der Krankheitsaktivität vor. Demnach sollen neben der Schubrate, der Verschlechterung körperlicher Beeinträchtigungen und MRT-Kriterien auch neuropsychologische Parameter wie Fatigue, Depressionen, Angststörungen und die Lebensqualität 
berücksichtigt werden. Das auf der Krankheitsaktivität aufbauende Schema (Ampelschema) soll dem Arzt helfen, frühzeitig ein Therapieversagen und eine Zunahme der Krankheitsaktivität zu erkennen und die Therapie entsprechend anzupassen (Stangel et al. 2015).

Ein standardisiertes und validiertes Vorgehen zur Bestimmung und Bewertung patientenindividueller Krankheitsaktivität existiert aber derzeit nicht.

Bei Zunahme der Krankheitsaktivität von mild/ moderat zu (hoch)aktiv, sollen die Patienten frühzeitig auf eine Behandlung mit Alemtuzumab, Fingolimod oder Natalizumab umgestellt werden (DGN 2014), da sowohl häufige als auch lang andauernde Schübe zu einer Degeneration von Nervenzellen, zu körperlich bleibenden Beeinträchtigungen und kognitiven Funktionseinschränkungen führen können (Summers et al. 2008).

Patienten mit einer hohen Schubfrequenz zu Beginn der Erkrankung haben ein erhöhtes Risiko einer raschen Verschlechterung und eines frühzeitigen Übergangs in eine SPMS im Vergleich zu Patienten mit weniger Schüben (Scalfari et al. 2014), sodass auch zu Beginn der Erkrankung eine hohe Krankheitsaktivität besteht und damit Medikamente für einen (hoch)aktiven Verlauf indiziert sein können. Der Schub ist eine primär klinische Diagnose. Die Krankheitsaktivität ist aber unabhängig von der klinischen Manifestation als Schub im MRT messbar (paraklinische Manifestation).

\subsubsection{Versorgungssituation}

\section{Studienlage}

Seit 2001 werden im Rahmen einer Datensammlung der Deutschen Multiple Sklerose Gesellschaft (DMSG) deutschlandweit Daten aus MS-Zentren, Schwerpunktpraxen und Kliniken unter anderem zu soziodemografischen Merkmalen, Verlaufsformen, Progression und Therapien von Patienten mit MS gesammelt. Aktuell sind 161 Zentren beteiligt. Insgesamt sind Datensätze von über 44.000 Patienten vorhanden (DMSG 2014). Die Identifizierung der Patienten erfolgt über die ärztlich gestellte Diagnose einer MS. Aufgrund der Struktur des Regis- ters werden dort ausschließlich Patienten aus den oben genannten Einrichtungen abgebildet, sodass sich die Auswertungen auf diese Patientenklientel beziehen (Flachenecker et al. 2008; Rommer et al. 2015).

Studien, die auf Abrechnungsdaten (Sekundärdaten) von Versicherten der gesetzlichen Krankenversicherung (GKV) basieren (Hapfelmeier et al. 2013, Höer et al. 2014), bilden unabhängig von der versorgenden Einrichtung alle abrechnungsrelevanten Inanspruchnahmen ab. Sie sind robust gegenüber einer Verzerrung (Bias) der Ergebnisse aufgrund der Selektion der Studienpopulation (Schubert et al. 2008). Die Identifikation der Patienten erfolgt über mindestens zwei Diagnosen einer MS, die durch Fachärzte der Neurologie, Nervenärzte oder Psychiater dokumentiert wurden oder über eine MS-spezifische Arzneimittelverordnung (ICD-10 GM G35,-, > Kap. 1). Allerdings können klinische Informationen wie Befunde bildgebender Verfahren, Laborwerte oder der Beeinträchtigungsgrad den Daten nicht entnommen werden. Dementsprechend liegen hier auch keine Informationen zur Krankheitsaktivität oder zur Krankheitsdauer der MS vor. Die Untersuchungen basierend auf GKV-Daten lassen auch keine Rückschlüsse über die Wirksamkeit und Nebenwirkungen zu. Da nur tatsächlich eingelöste Verordnungen in die Auswertungen eingegangen sind, bleibt unklar, in wie vielen Fällen Ärzte eine Verordnung zwar ausstellten, diese aber nicht eingelöst wurde. Ebenso ist es denkbar, dass Verlaufsformen, für die weniger bzw. keine medikamentösen Behandlungsansätze zur Verfügung stehen, in diesen Datensätzen unterrepräsentiert sind.

Daher sind die Verlaufsformen der MS zu unterschiedlichen Anteilen in den Studienpopulationen repräsentiert. In den Auswertungen der DMSG sind Patienten mit einer SPMS deutlich häufiger vertreten als in der Patientenpopulation basierend auf GKV-Daten (• Tab. 4.2).

Zum Zeitpunkt der Erstellung des Buches waren keine wissenschaftlichen Studien zur Inanspruchnahme der neu für die Therapie der MS zugelassenen Medikamente in Deutschland verfügbar. Die in den folgenden Abschnitten zitierten Erhebungen zur Adhärenz und zum Versorgungsbedarf aus Perspektive der Betroffenen und Behandelnden datie- 
- Tab. 4.2 Verteilung der Patienten mit MS differenziert nach den verschiedenen Verlaufsformen am Beispiel einer Patientenpopulation gesetzlich Versicherter im Vergleich zu Datenbasis der DMSG

\begin{tabular}{|c|c|c|}
\hline & Gesetzlich Versicherte Bayern & Datenbasis DMSG \\
\hline Autor & Höer et al. (2014) & Flachenecker et al. (2008) \\
\hline Erhebungszeitraum & $2005-2009$ & $2005-2006$ \\
\hline Datenbasis & Abrechnungsdaten & $\begin{array}{l}\text { übermittelte Datensätze von teilnehmen- } \\
\text { den Einrichtungen }\end{array}$ \\
\hline Zuordnung MS & $\begin{array}{l}\text { dokumentierte Diagnosen (ICD-10 GM } \\
\text { G35,-) oder ambulante Verordnung MS } \\
\text { spezifischer Arzneimittel }\end{array}$ & $\begin{array}{l}\text { ärztliche Diagnosen aller Verlaufsformen } \\
\text { aus der spezialisierten MS Versorgung }\end{array}$ \\
\hline $\mathrm{N}$ & $18.176(2009)$ & 5.445 \\
\hline Beobachtungszeitraum & $\begin{array}{l}\text { 2005-2009 } \\
\text { Tägliche Betrachtung, } \\
\text { jährliche Darstellung }\end{array}$ & $\begin{array}{l}\text { Querschnittsuntersuchung Stichtag } \\
31.12 .2006\end{array}$ \\
\hline \multicolumn{3}{|l|}{ Verlaufsform } \\
\hline Erstmanifestation (\%) & 10,2 & 1 \\
\hline KIS (\%) & k.A. & k.A. \\
\hline RRMS (\%) & 36,3 & 55 \\
\hline SPMS (\%) & 7,5 & 32 \\
\hline PPMS (\%) & 3,9 & 9 \\
\hline nicht spezifiziert (\%) & 38,9 & k.A. \\
\hline
\end{tabular}

ren ebenfalls vor der Verschreibungsfähigkeit der neu für die Therapie der MS zugelassenen Medikamente. Dem aktuellen Arzneimittelatlas lässt sich der Arzneimittelverbrauch in der Gesamt-GKV der letzten drei Jahre entnehmen (2012 bis 2014), sodass hier Informationen über die neuen Medikamente und die Marktdynamik bis 2014 einfließen (Häussler et al. 2015).

Der Barmer GEK Arzneimittelreport berichtet in einer Auswertung von Arzneimitteldaten der Jahre 2012 und 2013 unter anderem die Verordnungsanteile der für die Therapie der MS bis 2012 zugelassenen Medikamente ohne aber einen anteiligen Bezug zur Gesamtheit der MS-Patienten in der Versichertenpopulation der Barmer GEK oder einer Verlaufsform der MS herzustellen (Glaeske u. Schicktanz 2014).

\section{Allgemeine Inanspruchnahme}

In den folgenden Abbildungen ist der jährliche Verbrauch (gemessen in Tagesdosen) in der GesamtGKV von Arzneimitteln für die verlaufsmodifizierende Therapie bei MS inklusive der neu verfügbar gewordenen Ansätze dargestellt. Die Tagesdosis (engl. »defined daily dose«, DDD) ist die angenommene tägliche Erhaltungsdosis für die Hauptindikation eines Wirkstoffes bei Erwachsenen. Der Festlegung der DDD werden die Dosierungsempfehlungen gemäß den Fachinformationen zugrunde gelegt und auf 365 Tage umgerechnet. So wird unterschiedlichen Therapiezyklen Rechnung getragen, auch wenn die empfohlene Dosierung von einer täglichen Einnahme mit konstanter Dosis abweicht. Bei der DDD handelt es sich somit um eine Mengeneinheit, die in epidemiologischen Studien 


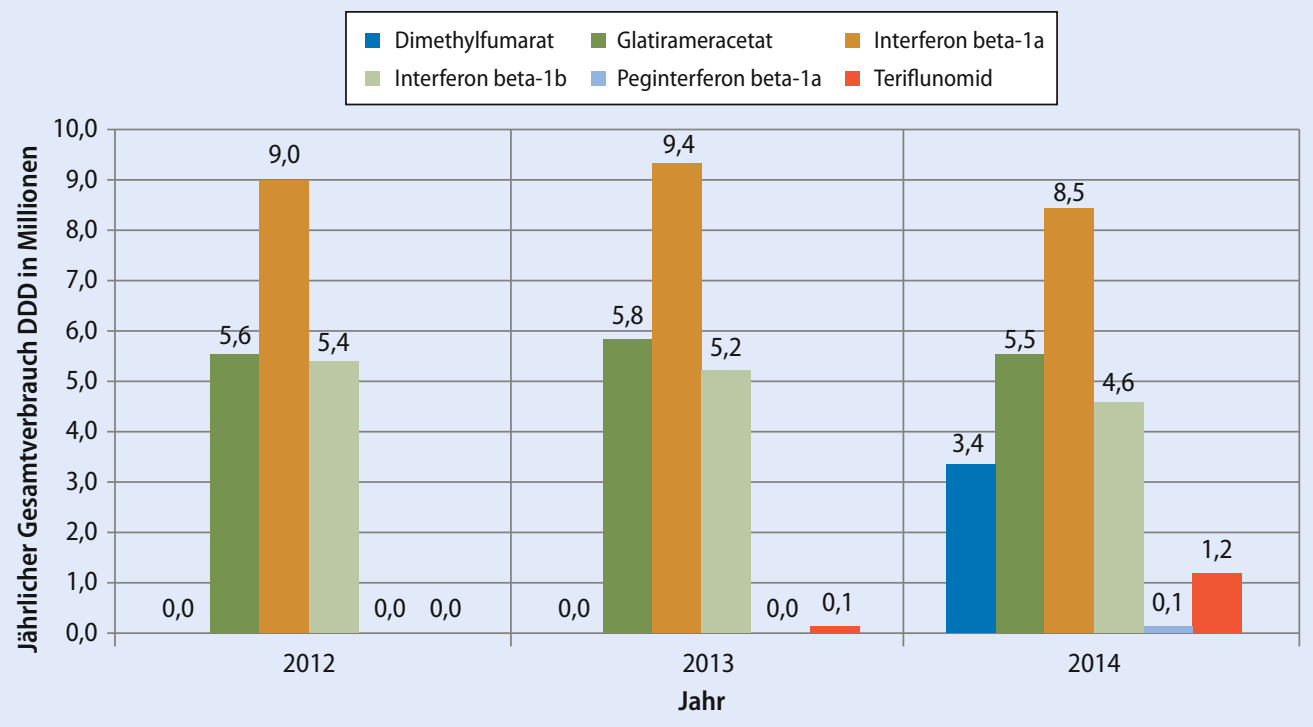

- Abb. 4.2 Jährlicher Verbrauch durchschnittlicher Tagesdosen (DDD) empfohlener Arzneimittel* für die verlaufsmodifizierende Therapie bei KIS, milden/moderaten Verläufen der RRMS oder bei SPMS mit aufgesetzten Schüben Anmerkung: *= zugelassene Indikationsgebiete siehe Leitlinie der DGN (2014). KIS = klinisch isoliertes Syndrom, RRMS = relapsing remitting MS, SPMS = secondary progressive MS. Marktzulassungen: Dimethylfumarat in 2014, Glatirameracetat in 2001, Interferon beta-1a in 1997, Interferon beta-1b in 1996, Peginterferon beta-1a in 2014, Teriflunomid in 2013 Quelle: IGES - Häussler et al. (2015)

den Verbrauch von Arzneimitteln auch über längere Zeiträume standardisiert erfassen soll. Auf diese Weise werden Verordnungen mit unterschiedlichen Wirkstärken, Packungsgrößen und Darreichungsformen vergleichbar (Fricke u. Beck 2014).

Die Betrachtung der in Anspruch genommenen Verbräuche der letzten drei Jahre in der GKV zeigt, dass die aktuellen Empfehlungen der DGN für die Therapie milder bis moderater und (hoch-)aktiver Verläufe grundsätzlich verfügbar sind und mit steigender Tendenz verordnet werden.

Bei Arzneimitteln zur Behandlung von milden bzw. moderaten Verläufen der MS stieg der Verbrauch in der GKV von 20,0 Mio. DDD im Jahr 2012 auf 23,3 Mio. DDD im Jahr 2014 (• Abb. 4.2). Dieser Anstieg war Folge dreier neuer Therapieoptionen in den Jahren 2013 und 2014. Im Oktober 2013 kam Teriflunomid auf den Markt und erreichte im Jahr 2014 einen Verbrauch von 1,2 Mio. DDD. Eine deutlich stärkere Marktdurchdringung erreichte der Fumarsäureester Dimethylfumerat. In den Monaten Mai bis Dezember 2014 wurden
3,3 Mio. DDD des Wirkstoffes abgegeben. Seit September 2014 steht eine pegylierte Form von Interferon-Beta-1a zur Verfügung (0,1 Mio. DDD im Jahr 2014) (Häussler et al. 2015).

Bei Arzneimitteln zur Behandlung von Verläufen der MS mit hoher Krankheitsaktivität war der Anstieg des Verbrauchs in der GKV von 2012 nach 2014 deutlich stärker (• Abb. 4.3). Im Jahr 2012 wurden 3,0 Mio. DDD abgegeben und im Jahr 2014 waren es 5,0 Mio. DDD. Insbesondere der Verbrauch des S1P-Rezeptor-Modulatoren Fingolimod nahm um mehr als das Doppelte von 1,4 Mio. DDD auf 3,1 Mio. DDD zu (Häussler et al. 2015).

Die Inanspruchnahme von Arzneimitteln für die verlaufsmodifizierende Therapie wird im Bereich zwischen $41,5 \%$ bis $71,0 \%$ der beobachteten Patienten mit MS beziffert (Flachenecker et al. 2008; Hapfelmeier et al. 2013; Höer et al. 2014). Diese Angaben beziehen sich auf Patienten ungeachtet der Verlaufsform oder der Krankheitsaktivität, sodass die Unterschiede unter anderem auch durch ein unterschiedliches Patientenkollektiv zu erklären sind. 


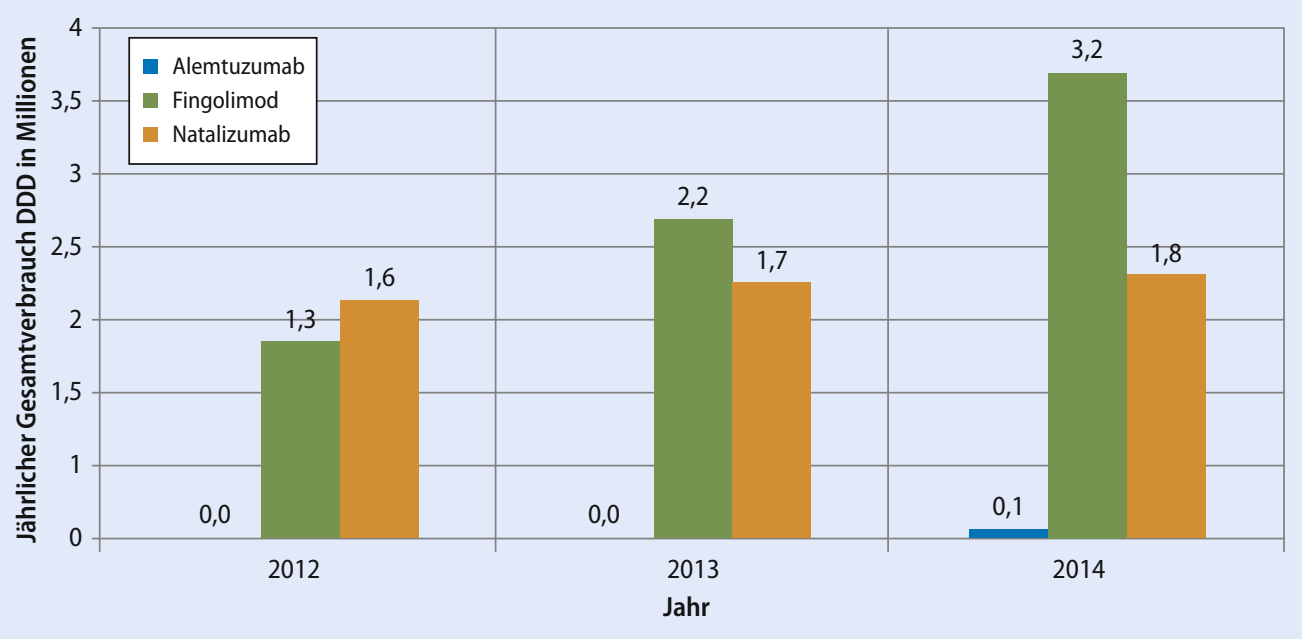

- Abb. 4.3 Jährlicher Verbrauch durchschnittlicher Tagesdosen (DDD) empfohlener Arzneimitteln* für die verlaufsmodifizierende Therapie bei (hoch-) aktiven Verläufen der RRMS Anmerkung: * zugelassene Indikationsgebiete siehe Leitlinie der DGN (DGN 2014). RRMS = relapsing remitting MS Marktzulassungen: Alemtuzumab in 2013, Fingolimod in 2011, Natalizumab in 2006 Quelle: IGES - Häussler et al. (2015)

Die Auswertung von Sekundärdaten gesetzlich Versicherter aus der Forschungsdatenbank der Gesundheitsforen Leipzig (GFL) zeigte, dass im Beobachtungszeitraum von 2006 bis 2010 41,5\% der Patienten mit MS (ICD-10 GM G35*) mindestens zwei Verordnungen eines Arzneimittels für die verlaufsmodifizierende Therapie erhielten (Interferon beta-1a, Interferon beta-1b, Glatirameracetat, Azathioprin, Mitoxantron oder Natalizumab) (Hapfelmeier et al. 2013).

Die Auswertung von Verordnungsdaten gesetzlich Versicherter $(\mathrm{n}=10.4$ Millionen) der Kassenärztlichen Vereinigung (KV) Bayerns ergab ein ähnliches Ergebnis. Höer et al. zeigten, dass 45,5\% (2005) bzw. 50,5 \% (2009) der Patienten mit einer von einem Facharzt für Neurologie, Nervenarzt oder Psychiater kodierten Diagnose einer MS pro Jahr mindestens eine Verordnung für eine verlaufsmodifizierende Therapie in Anspruch genommen haben. Berücksichtigt wurden Verordnungen für Interferon beta-1a, Interferon beta-1b, Glatirameracetat, Mitoxantron oder Natalizumab (nur in 2009). Der Anteil behandelter Patienten war in der Altersgruppe $<30$ Jahre am größten und nahm mit zunehmendem Alter ab. Über alle Altersgruppen nahm die Anzahl der eingelösten Verordnungen im
Beobachtungszeitraum zu (• Abb. 4.4) (Höer et al. 2014).

Eine Querschnittsuntersuchung von Patientendaten des DMSG-Datensatzes aus dem Zeitraum 2005/2006 ergab, dass $71 \%$ der beobachteten Patienten $(\mathrm{n}=3.431)$ eine verlaufsmodifizierende Therapie oder Glukokortikoide erhielten oder erhalten haben (Flachenecker et al. 2008). In dieser Untersuchung wurden Interferone (37,6\%) am häufigsten in Anspruch genommen (Flachenecker et al. 2008). Die Gabe von Glukokortikoiden zählt nicht zur verlaufsmodifizierenden Therapie und wurde bei den voran genannten Untersuchungen nicht mit betrachtet.

85,7 \% bzw. 87,6 \% der Verordnungen im Jahr 2009 für Interferone beta-1a bzw. Interferon beta- $1 \mathrm{~b}$ Präparate und 91,6 \% der Verordnungen für Natalizumab stellten Neurologen oder Nervenärzte aus (Höer et al. 2014).

Interferone und Glatirameracetat waren die am häufigsten verordneten Ansätze für die verlaufsmodifizierende Therapie (Hapfelmeier et al. 2013; Höer et al. 2014), wobei die Verordnungshäufigkeit im Beobachtungszeitraum 2005 bis 2009 für Interferone $\mathrm{ab}$ - und für Glatirameracetat zunahm (• Abb. 4.5). 


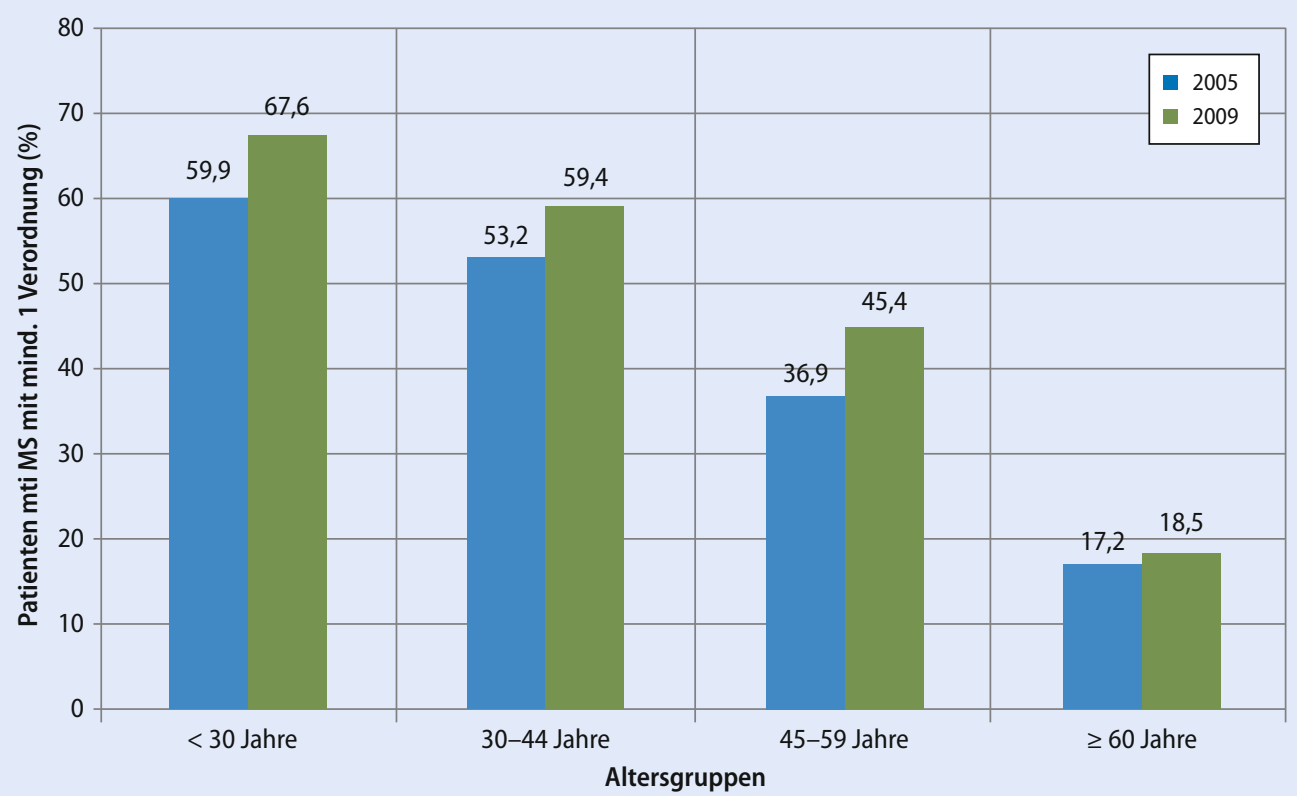

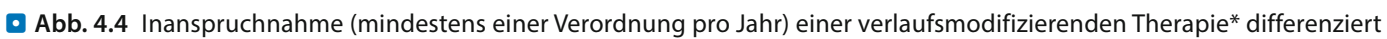
nach Altersgruppe Anmerkung: * = berücksichtigt wurden Verordnungen für Interferon beta-1a, Interferon beta-1b, Glatirameracetat, Mitoxantron oder Natalizumab (nur in 2009) Marktzulassungen: Glatirameracetat in 2001, Interferon beta-1a in 1997, Interferon beta-1b in 1996, Mitoxantron in 1985, Natalizumab in 2006

Quelle: IGES - Höer et al. (2014)

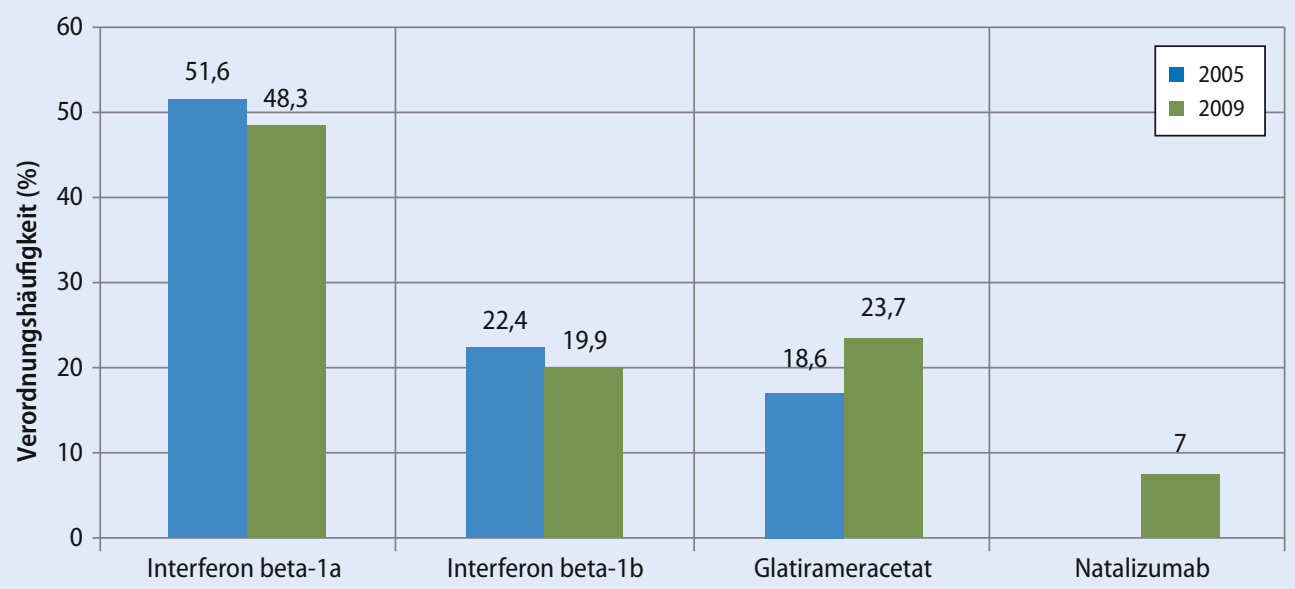

- Abb. 4.5 Verordnungshäufigkeit von Medikamenten für die verlaufsmodifizierende Therapie Anmerkung: Marktzulassungen: Glatirameracetat in 2001, Interferon beta-1a in 1997, Interferon beta-1b in 1996, Natalizumab in 2006 Quelle: IGES - Höer et al. (2014) 


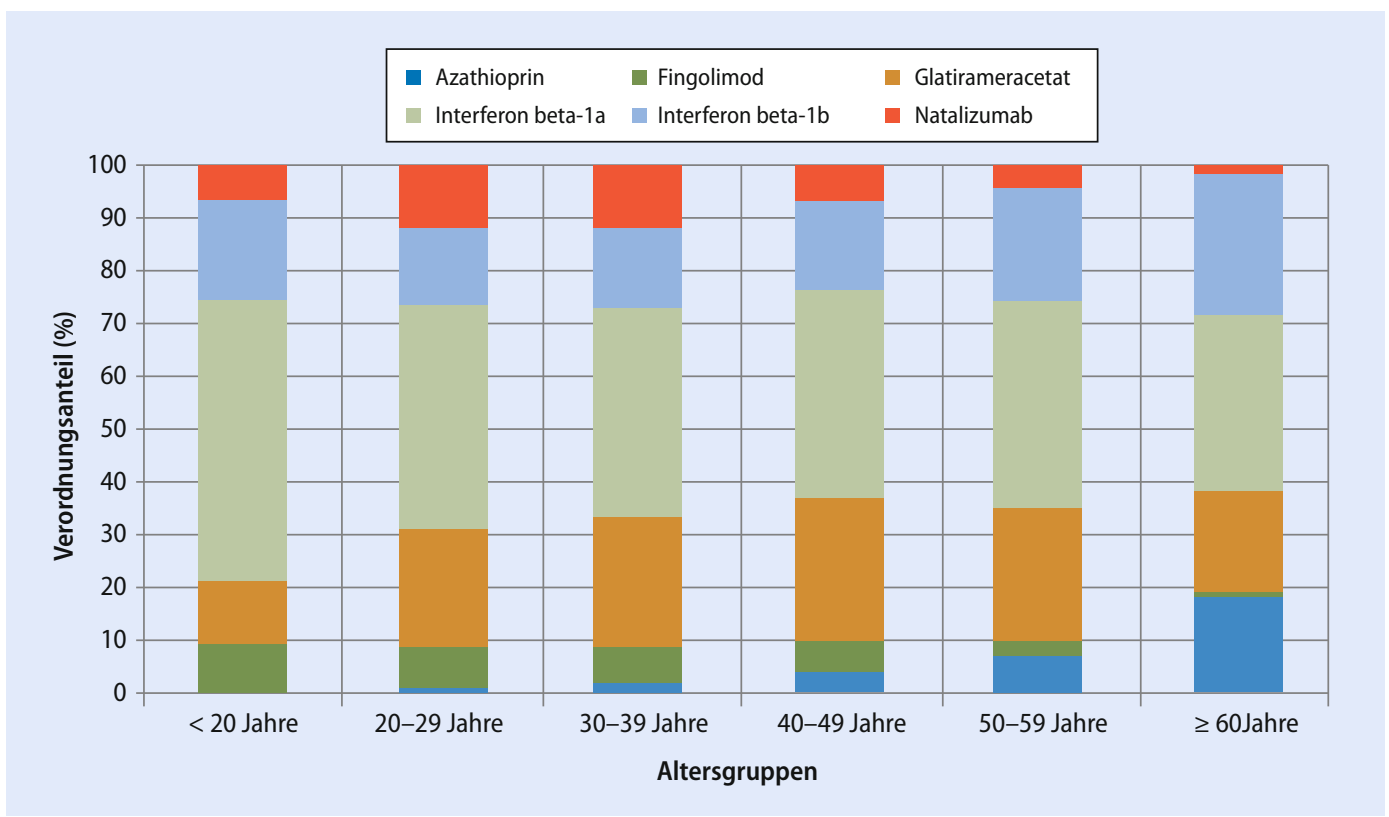

- Abb. 4.6 Verordnungsanteile von Medikamenten für die verlaufsmodifizierende Therapie bei gesetzlich Versicherten mit MS nach Altersgruppen (2012) Anmerkung: Marktzulassungen: Azathioprin in 1967, Fingolimod in 2011, Glatirameracetat in 2001, Interferon beta-1a in 1997, Interferon beta-1b in 1996, Natalizumab in 2006

Quelle: IGES - Glaeske u. Schicktanz (2014)

Der Arzneimittelreport der Barmer GEK untersuchte für ihre Versichertenpopulation für das Jahr 2012 die Verordnungsanteile von Medikamenten für die verlaufsmodifizierende Therapie unter behandelten Patienten mit MS ebenfalls nach Altersgruppen. In allen Altersgruppen waren Interferone die am häufigsten in Anspruch genommenen Medikamente, wobei der Anteil verordneter Interferon beta-1a-Präparate mit dem Alter ab und der Anteil verordneter Interferon beta-1b-Präparate zunahm. Der Verordnungsanteil von Glatirameracetat war in der Altersgruppe 40 bis 49 Jahre am größten. Der Anteil an Verordnungen für Natalizumab lag bei den 20- bis 29-Jährigen am höchsten. In der Altersgruppe 60 Jahre und älter waren es lediglich gut $1 \%$ der Verordnungen für die MS-Therapie, die auf diesen Wirkstoff fielen. Fingolimod wurde über alle Altersgruppen anteilig relativ konstant verordnet. Der Anteil lag bei den 20- bis 29-Jährigen bei $23 \%$, bei den 40 - bis 49 -Jährigen bei $27 \%$ und in der Altersgruppe $\geq 60$ Jahre bei $19 \%$. Azathioprin wurde in den jüngeren Altersgruppen kaum verordnet, in der Altersgruppe $\geq 60$ Jahre lag der Verordnungsanteil bei $18 \%$ (• Abb. 4.6).

\section{Inanspruchnahme verlaufsmodifizie- render Therapien in Abhängigkeit der Verlaufsform}

Aktuell stehen insbesondere verlaufsmodifizierende Therapien für die schubförmige MS zur Verfügung. Ein frühzeitiger Beginn wird empfohlen, um neue Schübe und Symptome zu vermeiden. Mit zunehmender Krankheitsdauer, Progredienz und sinkender Krankheitsaktivität nehmen die therapeutischen Möglichkeiten ab (DGN 2014). Die Verfügbarkeit therapeutischer Möglichkeiten spiegelt sich in der Inanspruchnahme differenziert nach Verlaufsform wider: Patienten mit RRMS nahmen im Vergleich zu Patienten mit progredienter Verlaufsform (SPMS, PPMS) deutlich häufiger eine verlaufsmodifizierende Therapie in Anspruch (• Abb. 4.7). 


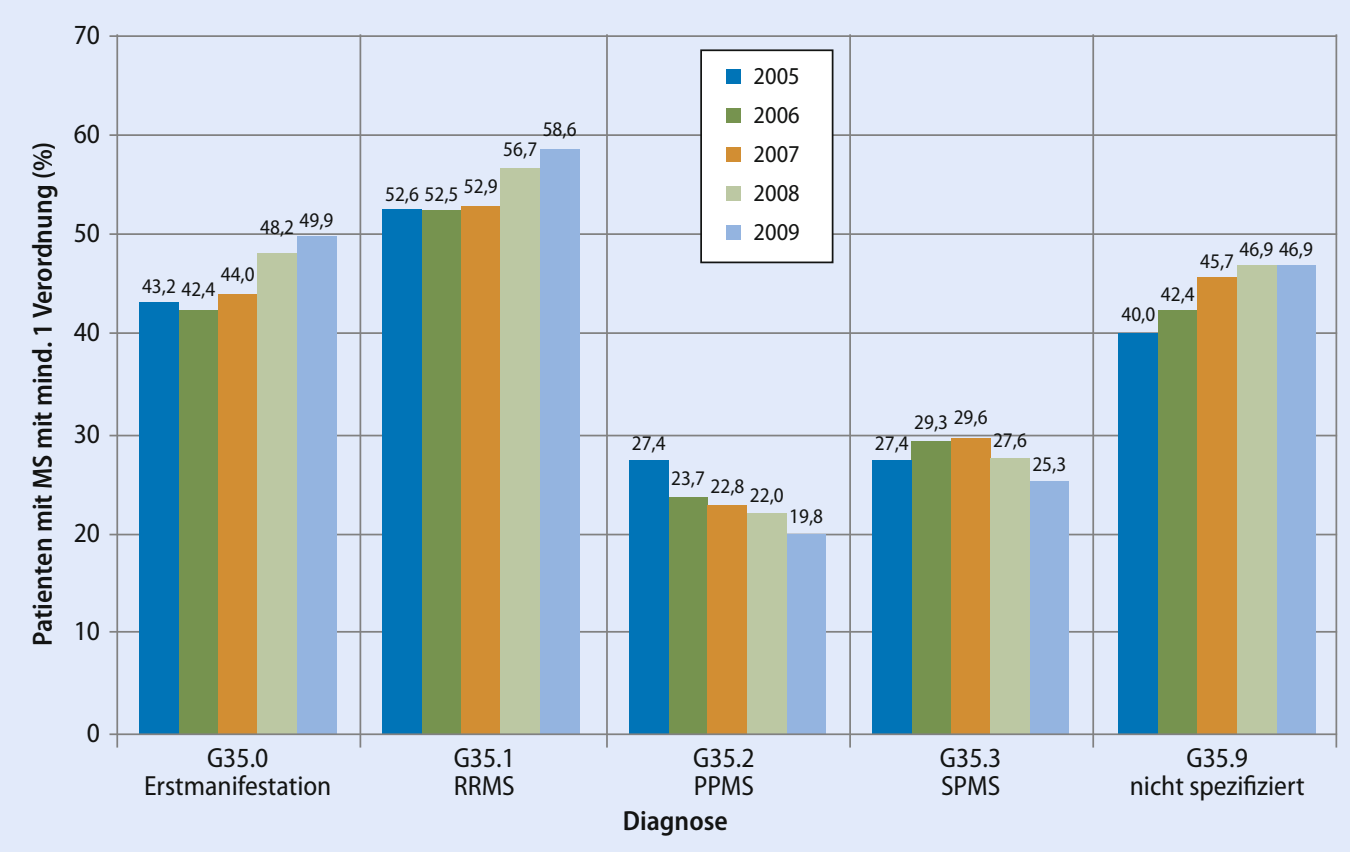

- Abb. 4.7 Anteil der Patienten mit MS mit mindestens einer Verordnung für eine verlaufsmodifizierende Therapie differenziert nach dokumentierter Verlaufsform der MS (ICD-10 GM G35,-) Anmerkung: RRMS = relapsing remitting MS, $S P S M=$ secondary progressive MS, PPMS = primary progressive MS

Quelle: IGES - Höer et al. (2014)

\section{- Versorgungssituation von Patienten mit Erstmanifestation}

Die Hälfte der Patienten mit einer von einem Facharzt der Neurologie diagnostizierten Erstmanifestation einer MS nahm im Jahr 2009 keine verlaufsmodifizierende Therapie in Anspruch (Höer et al. 2014). Der nach Diagnosestellung frühzeitige Therapiebeginn und die damit verbundene wirksame Reduzierung der Krankheitsaktivität und Schubprophylaxe sind aber für den weiteren Verlauf der Erkrankung prognostisch entscheidend. Insbesondere die Schubhäufigkeit in den ersten zwei Jahren nach Krankheitsbeginn und der Zeitraum zwischen dem ersten und zweiten Schub korrelieren mit dem körperlichen Beeinträchtigungsgrad im späteren Verlauf der Erkrankung (z.B. Scalfari et al. (2010)). Auch Patienten nach einem erstmalig aufgetretenen demyelinisierendem Ereignis (KIS) sollen daher Glatirameracetat oder Interferone, die derzeit für diese Indikation formal zugelassen sind, für die verlaufsmodifizierenden Therapie erhalten mit dem
Ziel, eine Konversion in eine RRMS oder SPMS zu verhindern (DGN 2014).

Die Dauer zwischen MS-Erstdiagnose bis zur nächsten fachärztlichen neurologischen (Neurologen, Nervenärzte und Psychiater) Behandlung variiert in Deutschland regional und ist abhängig von der Facharztdichte: eine Auswertung basierend auf Versichertendaten der Barmer GEK ergab, dass in Brandenburg (Facharztdichte: 4,4 pro 100.000 Einwohner) lediglich $37 \%$ der Patienten mit MS im Zeitraum von maximal sechs Wochen nach Erstdiagnose ambulant ärztlich durch einen Neurologen versorgt wurden, in Hamburg betrug dieser Anteil hingegen 63,5\% (Facharztdichte: 11,1 pro 100.000 Einwohner) (IGES Institut 2014).

Da über $90 \%$ aller MS-spezifischen Arzneimittelverordnungen durch Neurologen erfolgen und weniger als $1 \%$ dieser Patienten mit MS im Beobachtungsjahr ausschließlich durch Hausärzte betreut wurde (83,3 \% ausschließlich fachärztlich neurologisch) (IGES Institut 2014), ist davon auszuge- 
hen, dass die Mehrheit der nicht zeitnah nach Erstdiagnose versorgten Patienten auch nicht an anderer Stelle bezüglich ihrer MS-Erkrankung behandelt wurde.

\section{- Versorgungssituation von Patienten mit RRMS}

Gemäß aktuellem Update der Leitlinien für Diagnose und Therapie der MS sollen Patienten mit milden/moderaten Verläufen der RRMS neben Glatirameracetat oder Interferone, Dimethylfumarat oder Teriflunomid erhalten. Falls eine Therapie mit Interferonen nicht möglich ist oder unter einer bestehenden Behandlung mit Azathioprin ein stabiler Krankheitsverlauf besteht, kann Azathioprin theoretisch für die Therapie der MS weiter angewandt werden. Zur Neueinstellung gilt Azathioprin jedoch nicht mehr als Mittel der Wahl. Wenn es trotz Behandlung zu einer Zunahme klinisch relevanter oder messbarer Krankheitsaktivität kommt, wird ein Wechsel des Therapieansatzes empfohlen (- Abb. 4.1) (DGN 2014). Bei RRMS mit hoher Krankheitsaktivität werden als Mittel der Wahl Alemtuzumab, Fingolimod oder Natalizumab eingesetzt. Mitoxantron wird bei sekundär chronisch progredienter MS empfohlen und ist bei Versagen oder Unverträglichkeit anderer Immuntherapeutika zugelassen. Als letzte Therapieoption können mit experimentellen Verfahren individuelle Heilversuche unternommen werden (DGN 2014).

Höer et al. zeigten, dass 47,4 \% der Patienten mit RRMS im Jahr 2005 und 41,4\% der Patienten im Jahr 2009 überhaupt keine verlaufsmodifizierende Therapie erhielten (die seit dem Jahr 2009 neu für die MS zugelassenen Therapien wie Alemtuzumab, Dimethylfumarat, Fingolimod, Teriflunomid sind in der Untersuchung entsprechend nicht abgebildet) (Höer et al. 2014).

Die verlaufsmodifizierende Therapie hat nicht nur das Ziel, Schübe zu verhindern (Schubprophylaxe), sondern soll im besten Falle dazu führen, dass keine Krankheitsaktivität vorliegt ( $\triangleright$ Abschn. 4.1.2).

Auch bei Patienten mit »benignen" Verläufen, die sich zwar hinsichtlich der Zunahme körperlicher Beeinträchtigung über die Zeit von Patienten mit schweren Verlaufsformen unterscheiden, aber nicht im Mittel hinsichtlich der Abnahme der kognitiven Leistungsfähigkeit, ist eine (frühzeitige) dauerhafte verlaufsmodifizierende Therapie von ebenso großer Bedeutung (Gajofatto et al. 2015).

Eine retrospektive Auswertung zur Versorgungssituation von Patienten mit RRMS unter verlaufsmodifizierender Therapie $(n=7.896)$ für milde/moderate Krankheitsaktivität ergab, dass 33,6 \% der Patienten in den letzten 12 Monaten unter verlaufsmodifizierender Therapie einen Schub erlitten. Bei gut $8 \%$ der Patienten war mehr als ein Schub zu beobachten. Bei $61 \%$ der Patienten, die unter Therapie einen Schub erlitten, waren MRTDaten aus den vergangenen 12 Monaten vorhanden. Bei gut $90 \%$ dieser Patienten waren radiologische Zeichen hoher Krankheitsaktivität (über neun T2-Läsionen und/oder mindestens eine Gd-anreichernde Läsion) im MRT messbar. Die Autoren gaben an, dass bezogen auf alle Patienten somit bei gut einem Viertel der Patienten unter Therapie paraklinische Zeichen erhöhter Krankheitsaktivität messbar waren (Maurer et al. 2011), sodass diese eigentlich einer Therapie für (hoch)aktive Verlaufsformen zugeführt werden sollten.

Die behandelnden Ärzte dieser Untersuchung gaben an, bei $20 \%$ der Patienten eine Therapieumstellung erwogen $\mathrm{zu}$ haben. Klinisch relevante Parameter wie die Häufigkeit beobachteter Schübe waren besonders stark mit der Überlegung einer Therapieumstellung assoziiert, wohingegen messbare Kriterien erhöhter Krankheitsaktivität alleine im kranialen MRT weniger deutlich mit einer Therapieumstellung korrelierten (Maurer et al. 2011).

Flachenecker et al. berichteten, dass gut $15 \%$ der unbehandelten Patienten der DMSG-Datenbasis Schubaktivität zeigten und Zeichen einer raschen Progression der Erkrankung zu beobachten waren (Flachenecker et al. 2008). Eine Folgeuntersuchung des DMSG-Datensatzes (Stand 2009, $\mathrm{n}=8.695)$ ergab, dass die Wahrscheinlichkeit eines Therapieabbruchs mit der Krankheitsdauer und höherem körperlichem Beeinträchtigungsgrad zunahm (Khil et al. 2009). Die neu für die Therapie der RRMS zugelassenen Therapieansätze sind in den zitierten Studien nicht abgebildet, da sie zum Zeitpunkt der Datenerhebungen noch nicht zur Verfügung standen.

Eine erhöhte Schubrate ist Ausdruck einer nicht ausreichenden Schubprophylaxe und erhöhten Krankheitsaktivität unter Umständen aufgrund ei- 
ner medikamentösen Unterversorgung (Nichtinanspruchnahme) oder Fehlversorgung (Inanspruchnahme eines nicht ausreichend wirksamen Medikaments).

$14,6 \%$ der Patienten mit MS ( $\mathrm{n}=29.850)$ innerhalb der Barmer GEK Versichertenpopulation wurden 2012 wegen einer MS (Behandlungsdiagnose ICD-10 GM G35,-) stationär behandelt (Glaeske u. Schicktanz 2014). Der Großteil dieser im Krankenhaus behandelten Patienten war zwischen 40 und 60 Jahre alt und etwa zwei Drittel waren Frauen. 67,3 \% der im Krankenhaus behandelten Patienten (durchgängig versichert, keine stationäre Behandlung sechs Monate vor Krankenhauseinweisung in 2012, $\mathrm{n}=2.168)$ nahmen sechs Monate vor der stationären Aufnahme keine verlaufsmodifizierende Therapie in Anspruch. Bei 27,4\% dieser unbehandelten Patienten wurde aber nach dem stationären Aufenthalt eine verlaufsmodifizierende Therapie in der ambulanten Versorgung dokumentiert (Glaeske u. Schicktanz 2014). Bei gut $30 \%$ der Patienten erfolgte eine stationäre Aufnahme bei bestehender verlaufsmodifizierender Therapie. Da eine Zuordnung in die Verlaufsformen der MS, Informationen zur Krankheitsaktivität oder andere klinische Informationen zur Beurteilung einer vorliegenden Indikation für eine verlaufsmodifizierende Therapie oder Änderung einer bestehenden Therapie nicht vorliegen, kann ein kausaler Zusammenhang zwischen stationärer Behandlung und einer medikamentösen Unter-, Über- oder Fehlversorgung auf Basis der Versichertendaten aber nicht hergestellt werden (Glaeske u. Schicktanz 2014).

\section{- Verlaufsmodifizierende Therapie} bei progredienten Verlaufsformen

Die Wirksamkeit der verlaufsmodifizierenden Therapie nimmt mit Progression der Erkrankung ab. So stehen für die Behandlung der SPMS ohne aufgesetzte Schübe nur noch wenige therapeutische Optionen zur Verfügung. Für die Behandlung der PPMS besteht aktuell keine Empfehlung über einen wirksamen Therapieansatz. Der Anteil von Patienten mit SPMS, die eine verlaufsmodifizierende Therapie erhielten, betrug im Jahr 2009 25,3 \% bzw. $19,8 \%$ unter Patienten mit PPMS (Höer et al. 2014). Auf Basis der KV-Daten kann bei Patienten mit SPMS nicht zwischen Verläufen mit und ohne auf- gesetzte Schübe unterschieden werden. Der Anteil der Patienten mit progredienter Verlaufsform, die eine verlaufsmodifizierende Therapie erhalten, ist auch vor dem Hintergrund individueller Heilversuche zu bewerten.

Galushko et al. untersuchten in einer qualitativen Erhebung den Versorgungsbedarf von Patienten mit schwerer Verlaufsform der MS aus Perspektive der Betroffenen. Als dringlicher Bedarf wurde neben sozio-familiären Aspekten insbesondere der optimierte Zugang zu Einrichtungen der neurologischen Versorgung durch beispielsweise verbesserte Transportmöglichkeiten oder kürzere Wartezeiten sowie bessere Therapiemöglichkeiten identifiziert (Galushko et al. 2014).

Aus Perspektive der Leistungserbringer (Ärzte, Pfleger und Sozialarbeiter), die Patienten mit schweren Verläufen der MS betreuten, wurde ebenfalls ein barrierefreier Zugang zur medizinischen Behandlung sowie die Verfügbarkeit therapeutischer Optionen als wichtiger Versorgungsbedarf genannt (Golla et al. 2012).

\section{Adhärenz}

Die Adhärenz ist ein wichtiger Aspekt des Therapieerfolgs in der Behandlung der MS. Eine Zunahme der Adhärenz für verlaufsmodifizierende Arzneimittel ist mit einer geringeren Inanspruchnahme von Gesundheitsleistungen und Kosten in der Behandlung von Patienten mit MS assoziiert (Yermakov et al. 2015).

Der Terminus Adhärenz (»Einhaltung«) sollte zunächst von dem Begriff der Compliance abgegrenzt werden. Während Compliance (»Folgsamkeit«) sich auf das Ausmaß der Bereitschaft eines Patienten bezieht, medizinische Empfehlungen umzusetzen, wird unter Adhärenz die Übereinstimmung zwischen dem vereinbarten und dem tatsächlichen Patientenverhalten verstanden. Im Gegensatz zur Compliance liegt bei der Adhärenz folglich der Schwerpunkt auf einer Therapiepartnerschaft zwischen Patient und Arzt (Gorenoi et al. 2008).

Die Möglichkeiten zur Bestimmung der Adhärenz unterscheiden sich abhängig von der verwendeten Immuntherapie. Prinzipiell werden direkte (z.B. Bestimmung des Serumspiegels) von indirekten Methoden (z.B. über eingelöste Verordnungen) zur Bestimmung der Adhärenz abgegrenzt. 
In einer 2015 erschienenen retrospektiven Kohortenstudie wurde die Adhärenz bezüglich der verlaufsmodifizierenden Therapie unter Patienten mit MS ( $\mathrm{n}=50.057)$ in Deutschland untersucht. Basierend auf Abrechnungsdaten gesetzlich Versicherter, die dem Deutschen Arzneiprüfungsinstitut e.V. von gut $80 \%$ der Apotheken zur Verfügung standen, wurden im Zeitraum 2002 bis 2006 Patienten mit erstmaliger oder erneuter Therapie der damals verfügbaren Medikamente (Interferon beta-1a (im.), Interferon beta-1b (sc.) oder Glatirameracetat (sc.)) für 24 Monate (730 Tage) hinsichtlich adhärenter Inanspruchnahme (Compliance und Persistenz) beobachtet. Als Proxy für die Compliance diente die medication possession ratio (MPR), ein Verhältnis, das sich aus der Anzahl eingelöster DDD zum entsprechenden, definierten Zeitraum ergibt. Die Persistenz (kontinuierliche Einnahme) wurde als Anzahl der Tage nach erster Verordnung bis zum Aussetzen der Medikation um das Doppelte der Tage der vorangegangenen Therapiedauer ermittelt. Weniger als $40 \%$ der beobachteten Patienten nahmen die verlaufsmodifizierende Therapie an mindestens $80 \%$ des Beobachtungszeitraums von 24 Monaten überhaupt in Anspruch (Adhärenz). Bei zwei Drittel der Patienten wurde die Therapie für einen signifikanten Zeitraum unterbrochen, sprich lediglich gut $30 \%$ aller Patienten nahmen die empfohlenen Medikamente über einen Zeitraum von 24 Monaten auch kontinuierlich ein. $25 \%$ aller Patienten unterbrachen die Therapie innerhalb der ersten drei Monate nach Beobachtungsbeginn (Hansen et al. 2015).

Häufige Gründe einer niedrigen Adhärenz unter Patienten mit MS sind Nebenwirkungen wie Fieber oder Schmerzen sowie eine nachgewiesene oder angenommene fehlende Wirksamkeit der verlaufsmodifizierenden Therapie (Bischoff et al. 2012; Twork et al. 2007).

Frühe Abbrüche werden dabei im Zusammenhang mit Nebenwirkungen und späte (mehr als ein Jahr nach Therapiebeginn) Therapieabbrüche im Zusammenhang einer fehlenden (wahrgenommenen) Wirksamkeit diskutiert (Hansen et al. 2015).

Begleiterkrankungen wie Fatigue oder Depressionen erhöhen ebenfalls das Risiko, dass Patienten eine begonnene verlaufsmodifizierende Therapie abbrechen oder gar nicht erst beginnen (Bischoff et al. 2012; Tarrants et al. 2011).
Die parenterale Darreichungsform für die verlaufsmodifizierende Therapie wird von vielen $\mathrm{Pa}$ tienten als unangenehm empfunden und ist daher nicht selten ein Grund für einen Therapieabbruch. Ein großer Teil der Patienten gibt aber an, die Therapie mit einem anderen Präparat fortsetzen zu wollen (Bischoff et al. 2012).

Eine fehlende Krankheitseinsicht ist ein wichtiger Risikofaktor dafür, die verlaufsmodifizierende Therapie zu unterbrechen oder ganz abzubrechen. Auch bei Symptomlosigkeit ist bei mangelnder Information über mögliche Verläufe und Auswirkungen der Erkrankung eine effektive Schubprophylaxe erschwert. Aber gerade in der frühen Phase der Erkrankung besteht die Aussicht auf den größtmöglichen neuroprotektiven Effekt der verlaufsmodifizierenden Therapie.

Zur Erhöhung der Adhärenz bei Patienten mit MS werden zum einen Bestrebungen unternommen, den Patienten zu mehr Eigenverantwortung bezüglich seiner Erkrankung zu befähigen. Für dieses »Empowerment« werden Patientenschulungen als wichtiges Mittel genutzt, den Patienten hinsichtlich Risiken und Nebenwirkungen der verlaufsmodifizierenden Therapie und hinsichtlich der Risiken einer ausbleibenden Anwendung zu informieren. Der Grundgedanke dahinter ist, die Perspektive des Patienten in den medizinischen Entscheidungsfindungsprozess mit einzubeziehen. Dieses wird auch als partizipative oder informierte Entscheidungsfindung bezeichnet, bei der gegenseitig Informationen ausgetauscht und das Autonomiebedürfnis des Patienten berücksichtigt werden sollen (Heesen et al. 2006). Ein Großteil der Betroffenen und behandelnden Ärzte begrüßen eine autonome Beteiligung an den Therapieentscheidungen (Kopke et al. 2012).

Eine evidenzbasierte Patientenschulung (EBPS) unter Patienten mit MS in einem frühen Stadium der Erkrankung führte zu Zuwachs an Information hinsichtlich Risiken und Nutzen der verlaufsmodifizierenden Therapie ( $\triangleright$ Kap. 1.3). Die Inanspruchnahme und Haltung gegenüber der verlaufsmodifizierenden Therapie konnte im Vergleich zur Kontrollgruppe, die an keiner EBPS teilgenommen hatten, allerdings nicht verbessert werden (Kopke et al. 2014).

Zum anderen können Ärzte dazu beitragen, die Adhärenz zu erhöhen, wenn sie gemeinsam mit dem Patienten eine Therapie auswählen, die die in- 
- Tab. 4.3 Häufigste DRG in Zusammenhang mit einer Hauptdiagnose MS
DRG
Bezeichnung
B68D
MS und zerebellare Ataxie ohne äußerst schwere Komorbidität und Komplikationen (CC), 98 \% der Fälle dieser DRG mit Hauptdiagnose MS (ICD-10 GM G35,-)
B68A MS und zerebellare Ataxie mit äußerst schweren CC, 88 \% der Fälle dieser DRG mit Hauptdiagnose MS
(ICD-10 GM G35,-)

Quelle: IGES - DRG-Browser, Datenjahr 2013 (InEK 2015)

Anmerkung: Die Einheit der Abfrage ist der Fall. Einem Patienten können pro Jahr mehrere Fälle zugeordnet sein. B68D: $76 \%$ Normallieger, $18 \%$ Kurzlieger, $6 \%$ Langlieger.

B68A: $82 \%$ Normallieger, 7 \% Kurzlieger, $11 \%$ Langlieger.

dividuellen Lebensumstände und Präferenzen des Patienten berücksichtigen (Remington et al. 2013).

\section{Inanspruchnahme bei akutem Schub}

Der akute Schub ist eine klinische Manifestation erhöhter Krankheitsaktivität ( $>$ Kap. 3.2). Die Akuttherapie des MS-Schubes ist die Behandlung der Entzündungsreaktion mit einer relativ unspezifischen Hemmung des Immunsystems (GlukokortikoidStoßtherapie). Die Therapie des akuten Schubes beinhaltet im Wesentlichen die intravenöse Methylprednisolon-Pulstherapie, die eine bessere Wirksamkeit und geringere Nebenwirkungen gegenüber der oralen Glukokortiokoidtherapie hat. Bei Therapieversagen der Pulstherapie stehen die Plasmapherese oder die Immunadsorption mit dem Ziel der Verringerung der als ätiologisch ursächlich angenommenen Autoantikörper zur Verfügung (DGN 2014).

Die Pulstherapie könnte in Abwesenheit von schwerer Komorbidität und Komplikationen ambulant durchgeführt werden. Knapp jeder Dritte gesetzlich versicherte Patient (KV Bayern) mit einer MS (ICD-10 GM G35,-) erhielt im Jahr 2009 mindestens eine ambulante Verordnung für ein systemisches Glukokortikoid. 67,5 \% dieser Verordnungen wurden von Neurologen bzw. Nervenärzten verschrieben (Höer et al. 2014).

Bei 36 \% der Patienten des DMSG-Datensatzes, die im Zeitraum 2006 bis 2011 Glukokortikoide erhielten, wurde die Therapie des Schubs ambulant durchgeführt. Die Wahrscheinlichkeit einer ambulanten Behandlung nahm in dieser Untersuchung mit der Krankheitsdauer und dem Schweregrad körperlicher Beeinträchtigungen zu (Rommer et al. 2015).
Des Weiteren wurde der Einfluss von Patientencharakteristika auf den Gebrauch von Glukokortikoiden in der Behandlung von Patienten mit RRMS oder SPMS mit aufgesetzten Schüben untersucht. Es wurden die Patienten eingeschlossen, bei denen im Beobachtungszeitraum mindestens eine Behandlung mit Glukokortikoiden erfolgte $(n=5.106)$. Im ersten Beobachtungsjahr (2006) wurden $65 \%$ der beobachteten Patienten mit Glukokortikoiden für die Therapie des Schubs behandelt. Der Anteil sank auf ca. $55 \%$ im letzten Beobachtungsjahr (2011). Parallel dazu nahm der Anteil der Patienten, die Natalizumab erhielten, von $<2 \%$ auf $12 \%$ im Jahr 2009 $\mathrm{zu}$ (Rommer et al. 2015), sodass die Anwendung dieses Medikamentes unter Umständen den Rückgang mit beeinflusst hat.

Zur Therapie des Schubs und hochaktiver Phasen mit schneller Verschlechterung bzw. Progression der Erkrankung erfolgt in vielen Fällen die stationäre Aufnahme. Das Institut für das Entgeltsystem im Krankenhaus (InEK GmbH) stellt Daten abgerechneter stationärer Leistungen öffentlich mittels DRG-Browser zur Verfügung. Alle Krankenhäuser im Bundesgebiet sind zur Datenübermittlung verpflichtet. Dem Browser liegen die Daten aus der Datenlieferung gemä $\$ 21$ KHEntgG für das Datenjahr 2013 (Datenstand 31.05.2014), basierend auf der Gruppierung nach G-DRG Version 2013/2014, zugrunde (InEK 2015).

Die Darstellung von stationären Versorgungsleistungen für die Therapie des akuten Schubs erfolgt differenziert nach DRG spezifisch für die Behandlung der MS (•Tab. 4.3). 
- Tab. 4.4 Anteil der Verlaufsform unter Berücksichtigung der dokumentierten Krankheitsaktivität (akuter Schub, Exazerbation) in der stationären Versorgung der MS im Jahr 2013

\begin{tabular}{|c|c|c|c|c|c|}
\hline \multirow[b]{2}{*}{ ICD-10 GM } & \multirow[b]{2}{*}{ Beschreibung } & \multicolumn{2}{|c|}{$\begin{array}{l}\text { B68D, ohne CC } \\
(n=37.483)\end{array}$} & \multicolumn{2}{|c|}{$\begin{array}{l}\text { B68A, mit CC } \\
(n=419)\end{array}$} \\
\hline & & Fälle (n) & $\%$ & Fälle (n) & $\%$ \\
\hline G35.0 & Erstmanifestation & 4.712 & $12,57 \%$ & 0 & $0,00 \%$ \\
\hline G35.10 & RRMS & 2.835 & $7,56 \%$ & 15 & $3,58 \%$ \\
\hline G35.11 & RRMS, akuter Schub oder Exazerbation & 11.442 & $30,53 \%$ & 38 & $9,07 \%$ \\
\hline G35.20 & PPMS & 1.897 & $5,06 \%$ & 31 & $7,40 \%$ \\
\hline G35.21 & PPMS, akuter Schub oder Exazerbation & 2.114 & $5,64 \%$ & 48 & $11,46 \%$ \\
\hline G35.30 & SPMS & 5.042 & $13,45 \%$ & 61 & $14,56 \%$ \\
\hline G35.31 & SPMS, akuter Schub oder Exazerbation & 7.578 & $20,22 \%$ & 154 & $36,75 \%$ \\
\hline G35.9 & MS, nicht näher bezeichnet & 960 & $2,56 \%$ & 22 & $5,25 \%$ \\
\hline $\mathrm{G} 35^{*}$ & Gesamt & 36.580 & $97,59 \%$ & 369 & $88,07 \%$ \\
\hline G35. x1 & mit Schub oder Exazerbation & 21.134 & $56,39 \%$ & 240 & $57,28 \%$ \\
\hline
\end{tabular}

In über $55 \%$ der stationären Behandlungsfälle von Patienten mit MS war 2013 der akute Schub oder eine Exazerbation der Behandlungsgrund. Über die Hälfte der Fälle akuter Schübe unter Patienten mit MS ohne äußerst schwere Komorbidität und Komplikationen (CC) wurde als akuter Schub der RRMS klassifiziert. Unter Patienten mit äußert schweren CC war der Großteil auf einen akuten Schub oder eine Exazerbation einer SPMS oder PPMS zurückzuführen (•Tab. 4.4).

In $33 \%$ der stationären Fälle von MS ohne äußerst schwere und in $14 \%$ der stationären Fälle von MS mit äußerst schwerer Komorbidität und Komplikationen wurde eine Immuntherapie durchgeführt. Die am häufigsten durchgeführte Immuntherapie war die intravenöse Kortisontherapie. In 0,84 \% der Fälle von MS ohne äußerst schwere CC wurde eine Plasmapherese durchgeführt. In 0,45 \% der Fälle kam eine Immunadsorption zur Anwendung, bei 1,43\% der Fälle von MS mit äußerst schwerer CC wurde eine Immunadsorption dokumentiert (•Tab. 4.5).
Insgesamt wurden im Jahr 201321.134 Fälle eine MS und zerebellare Ataxie ohne äußerst schwere Komorbidität und Komplikationen (B68D) stationär wegen eines akuten Schubs oder einer Exazerbation behandelt. In 15.461 der Fälle wurde eine Therapie des Schubs oder Exazerbation dokumentiert (• Abb. 4.8).

Die Diskrepanz zwischen der Anzahl an Fällen mit Behandlungsdiagnose eines akuten Schubs und tatsächlich therapierten Schüben in der stationären Versorgung kann Hinweis darauf sein, dass ein Teil der Patienten ambulant weiterbehandelt werden konnte und wurde.

Eine wichtige Differenzialdiagnose des akuten Schubs ist eine durch einen Infekt oder Wärme ausgelöste Verschlechterung mit den klinischen Symptomen des Schubs. Diese ist aber nicht auf eine erhöhte Krankheitsaktivität zurückzuführen, sondern auf eine Reizung des vernarbten Hirngewebes und bedarf keiner Behandlung im Sinne einer Schubtherapie (inklusive Uhthoff-Phänomen) (DGN 2014). 
Tab. 4.5 Anteil durchgeführter Prozeduren (OPS) für die Therapie (hoch-) aktiver Phasen der MS in der stationären Versorgung ohne (B68D) und mit (B68A) äußerst schwerer CC der MS

\begin{tabular}{|c|c|c|c|c|c|}
\hline \multirow[b]{2}{*}{ OPS } & \multirow[b]{2}{*}{ Beschreibung } & \multicolumn{2}{|c|}{$\begin{array}{l}\text { B68D, ohne CC } \\
(n=37.483)\end{array}$} & \multicolumn{2}{|c|}{$\begin{array}{l}\text { B68A, mit CC } \\
(n=419)\end{array}$} \\
\hline & & Fälle (n) & $\%$ & Fälle (n) & $\%$ \\
\hline $8-547^{*}$ & Immuntherapien, gesamt & 12.634 & $33,71 \%$ & 58 & $13,84 \%$ \\
\hline $8-547 . x$ & Sonstige & 107 & $0,29 \%$ & 0 & $0,00 \%$ \\
\hline $8-547.31$ & $\begin{array}{l}\text { Immunsuppression, sonstige } \\
\text { Applikationsform }\end{array}$ & 272 & $0,73 \%$ & 6 & $1,43 \%$ \\
\hline $8-547.30$ & iv. Kortisontherapie & 10.087 & $26,91 \%$ & 42 & $10,02 \%$ \\
\hline $8-547.2$ & Mit Immunmodulatoren & 1.995 & $5,32 \%$ & 10 & $2,39 \%$ \\
\hline $8-547.1$ & Mit modifizierten Antikörpern & 59 & $0,16 \%$ & 0 & $0,00 \%$ \\
\hline $8-547.0$ & $\begin{array}{l}\text { Mit nicht modifizierten Anti- } \\
\text { körpern }\end{array}$ & 114 & $0,30 \%$ & 0 & $0,00 \%$ \\
\hline $8-820^{*}$ & Plasmapherese & 319 & $0,84 \%$ & 0 & $0,00 \%$ \\
\hline $8-821^{*}$ & Immunadsorption & 167 & $0,45 \%$ & 6 & $1,43 \%$ \\
\hline $\begin{array}{l}\text { 8-544.0 o. 8-543.51 о. } \\
8-543.31 \text { o. } 8.543 .11 \text { o. } 8.542^{*}\end{array}$ & Chemotherapie & 1.892 & $5,04 \%$ & 7 & $1,67 \%$ \\
\hline 6-003.f0 & Natalizumab & 229 & $0,61 \%$ & 0 & $0,00 \%$ \\
\hline $6-003^{*}$ & Rituximab & 220 & $0,59 \%$ & 0 & $0,00 \%$ \\
\hline
\end{tabular}

\section{Verlaufsmodifizierende Therapie bei Kinderwunsch und Schwangerschaft}

Frauen und Männern mit MS und einem Kinderwunsch muss nicht grundsätzlich von einer Schwangerschaft bzw. Vaterschaft abgeraten werden. Die Fertilität ist durch die Erkrankung selbst unbeeinflusst. Das Risiko für Nachkommen von Patienten mit MS, ebenfalls an einer MS zu erkranken, ist nur sehr leicht erhöht (Westerlind et al. 2014). Die Reproduktionsmedizin, d.h. die hormonelle Stimulation, sollte allerdings kritisch diskutiert werden, da es Hinweise für hormonell induzierte Schübe gibt (DGN 2014).

Aktuell stehen keine Therapieoptionen für die verlaufsmodifizierende Therapie zur Verfügung, die unbedenklich während einer Schwangerschaft eingenommen werden können. Die meisten der verfügbaren Ansätze sollten spätestens bei Bekannt- werden der Schwangerschaft abgesetzt werden. Für die Therapie des akuten Schubs wird während der Schwangerschaft der Steroidpuls empfohlen mit strengerer Indikationsstellung während des ersten Trimenons. Die Einschränkungen für die Gabe von Immuntherapeutika in der Schwangerschaft für die Schubprophylaxe bei schubförmiger MS sind allerdings nicht mehr für alle Therapeutika als absolute Kontraindikationen zu betrachten (DGN 2014). Ausgenommen davon sind aufgrund des embryotoxischen Potenzials die Wirkstoffe Alemtuzumab, Azathioprin, Fingolimod, Mitaxantron, Natalizumab oder Teriflunomid. Sie sollten schon Wochen bis Monate vor Eintritt einer Schwangerschaft von Frauen und Männer abgesetzt werden. Bei Alemtuzumab wird ein Abstand von vier Monaten zur letzten Gabe bei Patientinnen empfohlen. Eine Therapie mit Fingolimod kann von Männern mit 


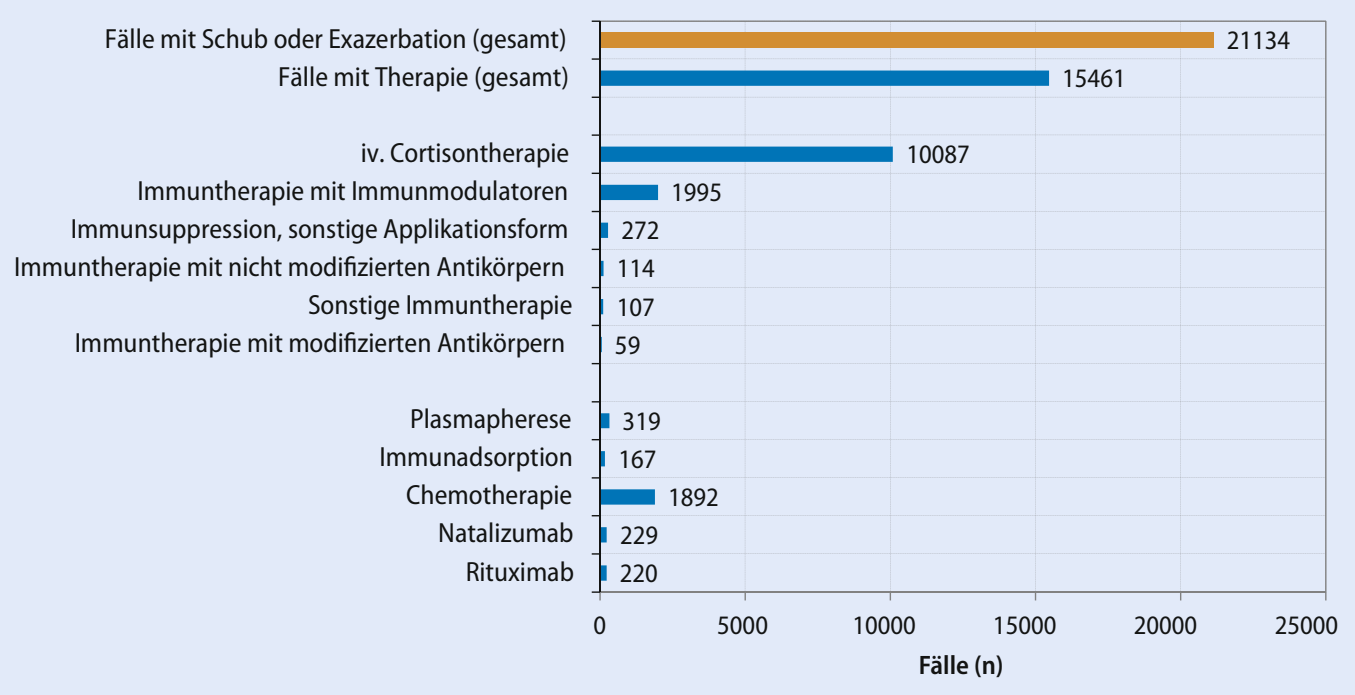

- Abb. 4.8 Anzahl stationärer Fälle mit Schub oder Exazerbation und Anzahl der Fälle mit dokumentierter Therapie (B68D, ohne (C) Anmerkung: CC = Komorbidität und Komplikationen, iv. = intravenös Quelle: IGES - DRG-Browser, Datenjahr 2013 (InEK 2015)

Kinderwunsch fortgeführt werden. Frauen wird davon abgeraten, die Therapie bis zum Eintritt der Schwangerschaft fortzusetzen. Bei Teriflunomid wird eine effektive Kontrazeption bis zum Abfall des Medikamentenspiegels unter 0,02 mg/l empfohlen sowie ein beschleunigtes Auswaschen im Falle einer ungeplanten Schwangerschaft. (DGN 2014). Eine prospektive Studie zur Frage erhöhter Abortneigung, Missbildungsrate, verringerter somatischer Entwicklung des Fötus und Frühgeburtlichkeit konnte keinen Zusammenhang zu väterlicher Einnahme von Interferon beta-Präparaten oder Glatirameracteat bei MS feststellen (Pecori et al. 2014).

Eine postnatale Schubprophylaxe durch intravenöse Immunglobuline (IVIG) während der Stillzeit wird weiterhin diskutiert. Nach Ende der Stillzeit sollte eher die zuvor etablierte Dauertherapie wiederaufgenommen werden, die Datenlage zu IVIG ist sehr schwach (DGN 2014).

Nach aktueller Datenlage basierend auf deutschlandweiten Daten des Multiple Sklerose und Kinderwunsch Registers (DMSKW) geht man davon aus, dass sich eine Schwangerschaft positiv auf den Verlauf der MS auswirken kann (Hellwig et al. 2008, 2012). Die zellulär vermittelte Entzündungsreak- tion bei MS wird durch den veränderten Immunstatus der schwangeren Frau unterdrückt (Wiendl et al. 2010) und die Schubhäufigkeit kann sich während der Schwangerschaft um bis zu $80 \%$ verringern (Hellwig et al. 2008). Nach der Entbindung ist das Risiko für Schübe für sechs Monate allerdings erhöht. Die (akzidentelle) Einnahme von Interferon beta-Präparaten oder Glatirameracetat während der Schwangerschaft reduzierte das Schubrisiko während und nach der Schwangerschaft im Vergleich zu unbehandelten Patientinnen (Hellwig et al. 2012).

Die Frauen sollten zum Stillen angeregt werden, da sich dies auch eher günstig auf die postpartale Schubrate auszuwirken scheint. Patientinnen, die ausschließlich stillten, hatten im Vergleich zu Müttern, die nicht stillten, eine deutlich geringere jährliche Schubrate $(0,68$ vs. 1,68$)$ (Hellwig et al. 2012).

Eine weitere Auswertung der Registerdaten schwangerer Frauen mit MS in Deutschland (DMSKW) zeigte, dass $90 \%$ der Frauen anfangen, ihre Kinder zu stillen, 30 \% davon mussten aber aufgrund der negativen hormonalen Wirkung bei notwendig werdenden Steroidpulsen die Brustmilchernährung beenden (Hellwig u. Gold 2008). 


\subsection{Symptomatische Therapie und Rehabilitation körperlicher und kognitiver Symptome}

Miriam Kip, Anne Talaschus, Iris-Katharina Penner

Die symptomatische Therapie hat das Ziel, die Auswirkungen der Erkrankung auf die körperlichen sowie kognitiven Fähigkeiten zu lindern und Folgekomplikationen zu vermeiden. Hierzu zählen auch die Abwendung psychosozialer Folgen und das Vermeiden dauerhafter Arbeitsunfähigkeit. Die Therapie soll sich dabei an dem individuellen Beschwerdebild und den Präferenzen der Betroffenen orientieren. Zentrales Element der symptomatischen Therapie ist die strukturierte multimodale (stationäre) Rehabilitation, bei der verschiedene Verfahren inklusive der Arzneimitteltherapie kombiniert Anwendung finden.

Insgesamt wird eine deutliche Unterversorgung in der symptomatischen Therapie festgestellt. Diese begründet sich zum einen in einem erschwerten $\mathrm{Zu}$ gang zu ambulant tätigen Kognitions- und Neuropsychologen oder Physiotherapeuten aufgrund fehlender Angebotskapazitäten. Zum anderen fehlen bei vielen Indikationen ausreichend wirksame, nicht-medikamentöse und medikamentöse Optionen sowie ärztliche Aufklärung und Informationen bei beispielsweise MS-assoziierten Störungen der Sexualität.

Auswertungen der Deutschen Multiple Sklerose Gesellschaft auf Basis von Daten aus den Jahren 2005/2006 zeigten, dass lediglich bei $17 \%$ bis $21 \%$ der Patienten mit Störungen der Kognition oder einer Fatigue und bei $63 \%$ der Patienten mit Depressionen eine Therapie dokumentiert war. Seit 2013 sind neuropsychologische Verfahren Teil des Gegenstandskatalogs der gesetzlichen Krankenversicherung. 2015 verzeichnete die Gesellschaft für Neuropsychologie 287 ambulant tätige Neuropsychologen im gesamten Bundesgebiet (2009 waren es 181). Aufgrund der geringen ambulanten Versorgungsdichte ist eine wohnortnahe Versorgung der Patienten derzeit nicht möglich. Aber gerade neuropsychologische Verfahren können die bereits im (frühen) Krankheitsverlauf auftretenden kognitiven Beeinträchtigungen oder Fatiguesymptome zuverlässig diagnostizieren und quantifizieren sowie eine depressive Symptomatik zeitnah aufdecken. Anschließende neurokognitive und psychologische Interventionen können helfen, die Krankheitsbewältigung und Adhärenz zu verbessern und so zu einer gesteigerten Teilhabe und Lebensqualität beitragen.

Physiotherapeutische Verfahren sind Mittel der ersten Wahl in der Behandlung motorischer Symptome und Einschränkungen der Mobilität z.B. aufgrund von Spastiken. Gut ein Drittel der im Datensatz der Deutschen Multiple Sklerose Gesellschaft registrierten $\mathrm{Pa}$ tienten erhielt keine Therapie der Spastik. $18 \%$ wurden nicht-medikamentös, $38 \%$ medikamentös und $13 \%$ in Kombination nicht-medikamentöser und medikamentöser Verfahren behandelt. Wenn physiotherapeutische Maßnahmen nicht genügen, die Symptomatik zu lindern oder zu stabilisieren, kann versucht werden, medikamentös eine Verbesserung zu erreichen. Aus der Perspektive von Patienten und Ärzten ist die Versorgungssituation der Spastik und assoziierter Schmerzen nicht zufriedenstellend, weil unter anderem wirksame und langfristig einsetzbare Arzneimittel derzeit fehlen. In einer bundesweiten Auswertung zur Behandlungssituation MS-induzierte Spastiken sahen die befragten Ärzte daher vor allen Dingen Potenzial im Ausbau physiotherapeutischer Verfahren.

Weitere Studien weisen auf eine zu geringe ärztliche Berücksichtigung sexueller Funktionsstörungen hin. Bei der Behandlung der sexuellen Dysfunktion besteht hier aus Perspektive der Betroffenen ein ungedeckter Aufklärungs- und Informationsbedarf.

Die MS ist mit einer Vielzahl unterschiedlicher Symptome assoziiert. Das Auftreten und die Ausprägung der Symptome können stark in der individuellen Krankheitsbiographie variieren. Phasen starker Symptomausprägung können sich mit Phasen schwacher oder gar stummer Ausprägung abwechseln. $\mathrm{Zu}$ den häufigsten und beeinträchtigendsten Phänomenen zählen neuropsychologische Symptome wie Störungen der Kognition, Fatigue, Depression, Angst, Spastik und andere Einschränkungen der Mobilität, Schmerzen sowie Blasenfunktionsstörungen und Störungen der Sexualität (DGN 2014). Manche Patientinnen und Patienten haben auch nach Jahren der Erkrankung kaum Beschwerden, während andere schon nach kurzer Krankheitsdauer unter deutlichen körperlichen und/oder neurokognitiven und neuropsychiatrischen Einschränkungen leiden. 
Die medizinische Rehabilitation ist das zentrale Element der symptomatischen Therapie. Sie folgt einem ganzheitlichen Ansatz und umfasst (neuro-) rehabilitative Verfahren, (neuro-) psychologische und psychotherapeutische Verfahren, Heil- und Hilfsmittel, Arzneimittel und andere Heilverfahren.

Im folgenden Kapitel werden verschiedene Therapieansätze sowie die Ziele der symptomatischen Therapie vorgestellt. Es folgt eine Einführung in die wichtigsten Prinzipien der symptomatischen Therapie, die multimodale (stationäre) Rehabilitation sowie die neuropsychologische Rehabilitation. Anschließend werden Versorgungssituation und Angebotskapazitäten beschrieben und symptombezogen aktuellen Empfehlungen der Versorgungsrealität gegenüber gestellt.

\subsubsection{Therapieziele und Übersicht empfohlener Therapieansätze}

Die symptomatische Therapie hat das Ziel, Auswirkungen der Erkrankung auf die körperlichen, geistigen und affektiven Gegebenheiten der Patienten zu verbessern und einer weiteren Verschlechterung entgegenzuwirken. Im Gegensatz zur verlaufsmodifizierenden Therapie vermag die symptomatische Therapie das Fortschreiten der Erkrankung selbst nicht $\mathrm{zu}$ beeinflussen. Übergeordnetes Ziel der symptomatischen Therapie ist, durch nicht-medikamentöse und medikamentöse Verfahren die soziale Teilhabe und Selbstbestimmung der Patienten $\mathrm{zu}$ fördern und damit eine Verbesserung der Lebensqualität zu erreichen (DGN 2014).

Im Vordergrund der symptomatischen Therapie steht die medizinische Rehabilitation. Sie hat das Ziel, den Auswirkungen der Erkrankung wie Beeinträchtigung von körperlichen und kognitiven Fähigkeiten und sozialen Beeinträchtigungen wie Behinderungen, Erwerbsunfähigkeit oder Pflegebedürftigkeit entgegenzuwirken (Augurzky et al. 2011).

$\mathrm{Zu}$ den rehabilitativen Maßnahmen in der Behandlung von Patienten mit MS zählen neuropsychologische Rehabilitationsverfahren, die Anwendung von Heilmitteln (Physiotherapie, Ergotherapie und Logopädie), Hilfsmitteln (z.B. Gehilfen) und Arzneimitteln. Im Rahmen der multimodalen (stationären) Rehabilitation kommen die verschiedenen
Verfahren kombiniert zur Anwendung (• Tab. 4.6, (DGN 2014; Beer et al. 2012)).

Medikamentöse Verfahren werden für die symptomatische Therapie allein oder in Kombination mit nicht-medikamentösen (rehabilitativen) Maßnahmen eingesetzt. In der Therapie der Spastik sind in den letzten Jahren neue Behandlungsoptionen hinzugekommen. So sind seit 2011 Cannabinoide bei schwerer Spastik als Behandlungsoption ( $\triangleright$ Kap. 6.1) und Fampridin für die (kurzfristige) Therapie der Spastik für Patienten mit MS zugelassen (•Tab. 4.7, (DGN 2014)).

\subsubsection{Prinzipien der Rehabilitation}

Gemäß der Internationalen Klassifikation der Funktionsfähigkeit (International Classification of Functioning, ICF) liegt der medizinischen Rehabilitation das Konzept der funktionalen Gesundheit zugrunde, das biologische, psychologische und soziale Komponenten berücksichtigt. Eine Indikation für die medizinische Rehabilitation ist bei Schädigungen der Körperfunktionen und -strukturen, Beeinträchtigungen der Aktivität und Beeinträchtigungen der Partizipation (Teilhabe) erfüllt (DGN 2012; World Health Organization 2005).

Die MS ist eine chronisch entzündlich-degenerative Erkrankung des ZNS. Die symptomatische Therapie basiert auf dem Prinzip der Rehabilitation und Prävention und richtet sich nach dem individuellen Beschwerdebild des Patienten. Da die Symptome häufig gemeinsam auftreten und sich gegenseitig bedingen oder verstärken können, eignen sich ganzheitliche bzw. multimodale Ansätze (DGN 2012). Aufgrund der Komplexität des Krankheitsbildes profitieren Patienten besonders von speziell in der Behandlung der MS geschultem und erfahrenem Personal (DGN 2014).

Bei nicht progredienten Verläufen tragen die Maßnahmen dazu bei, früh im Krankheitsverlauf Symptomen vorzubeugen, sie zu beseitigen oder zu verbessern. Bei Progression der Erkrankung gewinnen Maßnahmen der Symptomlinderung und Maßnahmen zur Abwendung einer wesentlichen Verschlechterung des Gesundheitszustandes des Patienten, z.B. durch Folgeerkrankungen und deren Komplikationen, an Bedeutung (Beer et al. 2012). Das 
- Tab. 4.6 Auswahl der wichtigsten Maßnahmen für die Rehabilitation von Patienten mit MS*

\begin{tabular}{|c|c|}
\hline Intervention & Beschreibung, Effekte Zielparameter \\
\hline Bewegungstherapie & $\begin{array}{l}\text { Verbesserung der Muskelkraft, Kondition und Beweglichkeit durch bei- } \\
\text { spielsweise Ausdauertraining und Widerstandstraining. Mechanisch } \\
\text { assistiertes Training kann Gehgeschwindigkeit und -strecke bei Patienten } \\
\text { mit schweren Einschränkungen der Gehfähigkeit verbessern. Linderung der } \\
\text { Fatiguesymptomatik. }\end{array}$ \\
\hline $\begin{array}{l}\text { Ergotherapie und Patienten- } \\
\text { schulungen }\end{array}$ & $\begin{array}{l}\text { Verbesserung motorischer und kognitiver Fähigkeiten, der Bewältigung } \\
\text { von Aufgaben des täglichen Lebens und der Kompensation. Reduktion der } \\
\text { Auswirkungen der Fatigue bei Patienten mit Einschränkungen alltäglicher } \\
\text { Aktivitäten. }\end{array}$ \\
\hline $\begin{array}{l}\text { Multimodale (multidisziplinäre) } \\
\text { Rehabilitation inklusive Heilmittel (z.B. } \\
\text { Physiotherapie, Logopädie) und } \\
\text { Hilfsmittel,(z.B. Kühlwesten, Gehhilfen) }\end{array}$ & Verbesserung der allgemeinen Funktionalität, Teilhabe und Lebensqualität. \\
\hline Ambulant & $\begin{array}{l}\text { Insbesondere für Patienten mit geringen bis mittleren Funktionsein- } \\
\text { schränkungen. }\end{array}$ \\
\hline Stationär & $\begin{array}{l}\text { Insbesondere für Patienten mit mittleren bis schweren Funktionsein- } \\
\text { schränkungen. }\end{array}$ \\
\hline Neuropsychologische Verfahren & $\begin{array}{l}\text { Verbesserung kognitiver Teilleistungen wie Aufmerksamkeit, Gedächtnis, } \\
\text { Geschwindigkeit, Multi-Tasking bei Patienten mit kognitiven Beeinträchti- } \\
\text { gungen. }\end{array}$ \\
\hline
\end{tabular}

Setting und die Auswahl der Verfahren erfolgen aufgrund des heterogenen Beschwerdebildes und der unterschiedlichen Krankheitsverläufe patientenindividuell in Abhängigkeit der funktionellen Einschränkungen. Auch die Festlegung realistischer Rehabilitationsziele erfolgt am sinnvollsten unter Berücksichtigung der individuellen Krankheitsbiografie und der persönlichen Präferenzen der Betroffenen (Beer et al. 2012).

Die Berücksichtigung individueller Determinanten, Ressourcen und Ziele sowie von Umweltfaktoren ist ein wichtiger Faktor dafür, dass rehabilitative Maßnahmen und psychotherapeutische Begleitung auch dauerhaft erfolgreich umgesetzt werden können (DGN 2012).

\section{Multimodale (stationäre) Rehabilitation}

Unter multimodaler Rehabilitation wird die kombinierte Anwendung verschiedener Interventionen verstanden (•Tab. 4.7). Eine systematische Über- sichtsarbeit zur Wirksamkeit von strukturierten multimodalen (multidisziplinären) ambulanten und stationären Rehabilitationsprogrammen unter erwachsenen Patienten mit MS ergab starke Belege für eine Steigerung des Aktivitätsniveau und der gesellschaftlichen Teilhabe von Patienten mit MS. Allerdings konnten keine Veränderungen auf Ebene der eigentlichen funktionellen Einschränkungen gefunden werden (Khan et al. 2007).

Eine 2015 erschienene systematische Übersichtsarbeit untersuchte die Wirksamkeit rehabilitativer Maßnahmen, die mittels Telemedizin dem Patienten zugänglich gemacht wurden. Durch das telemedizinische Angebot soll die Zugänglichkeit von Rehabilitationsverfahren erhöht werden. Gleichzeitig ist aber vorauszusetzen, dass die Patienten ausreichend Kapazitäten besitzen, die übermittelten Komponenten auch selbstständig durchzuführen oder sie jemanden haben, der sie dabei unterstützt (mobile Rehabilitation). Die Analyse deutete auf eine kurz- 
- Tab. 4.7 Übersicht der derzeit empfohlenen Arzneimittel für die symptomatische Therapie bei MS

\begin{tabular}{|c|c|}
\hline Arzneimittel & Beschreibung \\
\hline Therapie kognitiver Störungen & Aktuell keine Empfehlung, die auf nachgewiesener Evidenz basiert \\
\hline \multicolumn{2}{|l|}{ Therapie der Fatigue* } \\
\hline Amantadin & $\begin{array}{l}\text { M2-lonenkanalblocker. Steigert u.a. Dopaminfreisetzung. Darreichung oral. } \\
\text { Markteinführung } 1966 \text { (Gemeinsamer Bundesausschuss (G-BA) schloss } 2011 \text { Off- } \\
\text { Label-Use für MS explizit aus) }\end{array}$ \\
\hline Modafinil & $\begin{array}{l}\text { Einsatz bei Narkolepsie. Wirktyp wie Amphetamin, aber chemisch abweichend. } \\
\text { Genauer Wirkmechanismus nicht bekannt. Darreichung oral. Markteinführung } 1998\end{array}$ \\
\hline Antidepressive Therapie & Siehe Leitlinie (DGPPN et al. 2009) \\
\hline Schmerztherapie & $\begin{array}{l}\text { Siehe u.a. Leitlinien für Diagnostik und Therapie in der Neurologie: Pharmakologisch } \\
\text { nicht interventionelle Therapie chronisch neuropathischer Schmerzen (DGN 2015) }\end{array}$ \\
\hline \multicolumn{2}{|l|}{ Therapie der Spastik* } \\
\hline Baclofen & $\begin{array}{l}\text { Gamma-Aminobuttersäure (GABA)-Derivat (Neurotransmitter). Zentrales Muskelrela- } \\
\text { xans, stimuliert die GABA-Rezeptoren vorwiegend im Rückenmark und dämpft } \\
\text { dadurch die Erregungsübertragung. Darreichung oral oder parenteral (intrathekal). } \\
\text { Markteinführung } 1971\end{array}$ \\
\hline Benzodiazepine & $\begin{array}{l}\text { Muskelrelaxantien und Tranquilizer, verstärken dämpfende Wirkung des Neurotrans- } \\
\text { mitter Gamma-Aminobuttersäure (GABA). Hauptsächlich angstlösend und schlafan- } \\
\text { stoßend. Entsprechend auch muskelrelaxierend. Darreichung oral. Markteinführung } \\
\text { des ersten Wirkstoffs } 1960\end{array}$ \\
\hline Botulinum-Toxin A & $\begin{array}{l}\text { Neurotoxin. Hemmt Freisetzung von Azetylcholin. Entspannung des hyperaktiven } \\
\text { Blasenwandmuskels. Darreichung parenteral. Markteinführung } 1993 \text { (Seit } 2011 \\
\text { zugelassen für die Behandlung von Harninkontinenz bei MS) }\end{array}$ \\
\hline Cannaboide & $\begin{array}{l}\text { Tetrahydrocannabinol-Extrakt (THC) aus der Hanfpflanze. Betäubungsmittel. Dar- } \\
\text { reichung oral (Spray). Als Arzneistoff Nabiximols seit } 2011 \text { bei schwerer Spastik unter } \\
\text { MS zugelassen }\end{array}$ \\
\hline Dantrolen & $\begin{array}{l}\text { Kalziumkanalblocker, Wirkung auf das longitudinale System. Darreichung oral. } \\
\text { Markteinführung } 1978\end{array}$ \\
\hline Fampridin & $\begin{array}{l}\text { Kaliumkanalblocker, erhöht damit indirekt die Ausschüttung von Acetylcholin. } \\
\text { Verbesserung der Gehfähigkeit bei MS. Darreichung oral. Markteinführung } 2011\end{array}$ \\
\hline Gabapentin & $\begin{array}{l}\text { Gamma-Aminobuttersäure (GABA)-Derivat (Neurotransmitter). Erhöht die Frei- } \\
\text { setzung des GABA-Neurotransmitters. Darreichung oral. Markteinführung } 1995\end{array}$ \\
\hline Tizanidin & $\begin{array}{l}\text { Alpha2-Rezeptoragonist, zentral am Rückenmark wirkendes Skelettmuskelrelaxans. } \\
\text { Darreichung oral. Markteinführung } 1985\end{array}$ \\
\hline Tolperison & $\begin{array}{l}\text { Muskelrelaxans. Dämpfende Wirkung über das Zentralnervensystem. Darreichung } \\
\text { oral. Markteinführung } 1981 \text { (nicht für MS zugelassen) }\end{array}$ \\
\hline \multicolumn{2}{|l|}{ Therapie Ataxie und Tremor } \\
\hline Topiramat & $\begin{array}{l}\text { Sulfamat-substitutiertes Monosaccharid. Wirkmechanismus weitgehend unbekannt. } \\
\text { Dämpfende Wirkung über das Zentralnervensystem. Darreichung oral. Markteinfüh- } \\
\text { rung } 1997\end{array}$ \\
\hline
\end{tabular}


- Tab. 4.7 (Fortsetzung)

Arzneimittel

Beschreibung

Therapie von Blasenstörungen*

Anticholinergika

Wirkstoffe zur Unterdrückung von Acetylcholin im parasympathischen Nervensystem. Darreichung überwiegend oral aber auch extern (Pflaster). Dämpfen bei MS einen überaktiven Blasenmuskel. Markteinführung des ersten Wirkstoffs 1968

Botulinum-Toxin A

Toxin des Bakteriums Clostridium botulinum, lähmt periphere Muskeln durch irreversible Hemmung der Freisetzung von Acetylcholin. Darreichung parenteral. Markteinführung 1993

Therapie der sexuellen Dysfunktion*

Bei erektiler Dysfunktion: Phosphodiesterase-5-Inhibitoren. Darreichung oral. Markteinführung Sildenafil

Sildenafil, Tadalafil, Vardenafil 1998, Tadalafil 2003, Vardenafil 2003

Quelle: IGES - atd arznei-telegramm Arzneimitteldatenbank (2015); DGN (2014); Häussler et al. (2014); Mutschler et al. (2013); Schwabe u. Paffrath (2014)

Anmerkung: * =in alphabetischer Reihenfolge

fristige und langfristige Verbesserung körperlicher Einschränkungen sowie anderer Symptome wie der Fatigue durch telemedizinisch vermittelte Rehabilitationsverfahren hin (Khan et al. 2015).

Insgesamt besteht zunehmend Evidenz dafür, dass die multimodale Rehabilitation für die Betroffenen sinnvoll und empfehlenswert ist, da sie zu einer Steigerung der Aktivität sowie der gesellschaftlichen Teilhabe führen kann. Der kombinierte und auf den Patienten zugeschnittene Einsatz verschiedener Verfahren im Rahmen der multimodalen Therapie ist im Hinblick auf die Erbringung eines Wirksamkeitsnachweises jedoch herausfordernd, da dies den Einsatz komplexer Studiendesigns erfordert (Campbell et al. 2000). Der Grad der Evidenz bisheriger Studien ist daher oftmals als eher gering einzustufen.

\section{Physiotherapie, Bewegungstherapie und Sport (Heilmittel)}

Die Physiotherapie ist Kernkomponente der symptomatischen Therapie und Rehabilitation (Vogel 2015). Die Leitlinien der DGN verweisen im Rahmen der symptomatischen Behandlung der MS bei einer Vielzahl von Indikationen auf die Physiotherapie als eine sinnvolle Maßnahme. Symptome, welche effektiv behandelt werden können, sind beispielsweise zentrale Lähmungen, Koordinationsstörun- gen und Gleichgewichtsstörungen. Sie ist gemäß den Leitlinien die erste Wahl bei der Behandlung von Spastiken und wird ebenfalls bei Tremor und Ataxie empfohlen. Aber auch die Verbesserung von Störungen der Tiefensensibilität, Schwindel sowie Blasenstörungen können Ziele einer physiotherapeutischen Intervention sein (DGN 2014; DMSG Berlin e.V. 2015).

Körperliche Aktivitäten bei MS wurden lange Zeit mit unerwünschten Auswirkungen auf den Krankheitsverlauf in Verbindung gebracht. Entsprechend sollten sich die Patienten schonen und es wurde Bettruhe in den aktiven Krankheitsphasen verordnet. Kritisch hinterfragt wurde dieses Therapiekonzept bereits Mitte des 20. Jahrhunderts. Internationale Studien konnten zeigen, dass Bewegung keinen negativen Effekt auf den Krankheitsverlauf hat. Dennoch hielt sich die Behandlungsstrategie bis in die 90er-Jahre in Deutschland (Tallner et al. 2013).

Erste Belege dafür, dass Bewegung nicht nur unschädlich ist, sondern sich auch positiv auf den Krankheitsverlauf auswirken kann, wurden bereits in einer Studie von Russel und Palfrey in den 1960ern erbracht. In der Studie wurden 69 Patienten mit MS zwei Jahre beobachtet. Ihnen wurde ihm Rahmen einer stationären Reha ein Bewegungsprogramm erstellt, welches sie auch nach Entlassung fortführen 
und in Abhängigkeit von ihrer Konstitution um weitere sportliche Aktivitäten ergänzen sollten. Insgesamt zeigten sich bei keinem Teilnehmer negative Auswirkungen. 41 Patienten wiesen klinische Verbesserung auf, bei 28 Patienten stellte sich kein nachweisbarer Effekt ein (Russel u. Palfrey 1969).

Obwohl ein positiver Effekt körperlicher Bewegung heutzutage als allgemein anerkannt gilt, liegen dennoch nur wenige Studien vor, welche die konkreten Auswirkungen und Therapieempfehlungen beschreiben (Tallner et al. 2013). Dennoch kamen Latimer-Cheung et al. 2013 in ihrer Meta-Analyse, welche 54 Studien umfasste, zu dem Ergebnis, dass für Patienten mit einem milden bis moderaten Verlauf der MS eine positive Auswirkung von sportlichem Training auf die Muskelkraft und auf die aerobe Kapazität mit ausreichender Evidenz belegt ist. Zudem kamen sie zu dem Schluss, dass weiterhin die Mobilität, die Fatigue sowie die gesundheitsbezogene Lebensqualität verbessert werden können (Latimer-Cheung et al. 2013). Eine Metanalyse von Pearson et al. (2015, n=13 Studien) zeigte ebenfalls, dass sportliches Training bei Patienten mit MS eine Verbesserung der Gehgeschwindigkeit und der Ausdauer zu Folge hat (Pearson et al. 2015).

Von besonderer Wichtigkeit ist es, die Physiotherapie individuell an den Patienten anzupassen, um so seinem Krankheitsverlauf, welcher starken Schwankungen unterliegen kann, gerecht zu werden. Auch die jeweilige Tagesverfassung muss bei den therapeutischen Maßnahmen berücksichtigt werden (DMSG Berlin e.V. 2015).

Speziell für die MS finden verschiedene Therapiekonzepte Anwendung, welche durch physikalische Therapien, Lymphdrainage oder Stand- und Gangschulung ergänzt werden können. Genannt seien beispielsweise das Bobath-Konzept, die VojtaTherapie und die Propriozeptive Neuromuskuläre Fazilitation (PNF) (Simonow 2015).

Die genannten Therapiekonzepte sind Teil der Rahmenempfehlungen zur Heilmittelversorgung gemäß $\$ 125$ Abs. 1 SGB V und dürfen nur durch speziell geschulte Physiotherapeuten ausgeführt werden (Spitzenverbände der Krankenkassen et al. 2006).

\section{Neuropsychologische Rehabilitation}

Neuropsychologische Verfahren werden im Rahmen der stationären Neurorehabilitation seit vielen
Jahren angewandt (Gesellschaft für Neuropsychologie (GNP) e.V. 2013). Seit dem 1.1.2013 sind neuropsychologische diagnostische und therapeutische Verfahren auch ambulante Leistung der GKV (KV Hessen 2014). Basierend auf einem Beschluss des Gemeinsamen Bundesausschusses (G-BA) vom 24.11.2011 wurde die Richtlinie $\mathrm{zu} » U n t e r s u-$ chungs- und Behandlungsmethoden der vertragsärztlichen Versorgung « um diagnostische und therapeutische Verfahren ergänzt (Bundesministerium für Gesundheit 2012). Bei Vorliegen einer von einem Facharzt für Neurologie, Nervenheilkunde, Psychiatrie und Psychotherapie gestellten Diagnose (somatische Abklärung, Stellen der Indikation; - Tab. 4.8) kann die neuropsychologische Diagnostik und Therapie im weiteren Verlauf neben den oben genannten Fachgruppen ebenso durch ärztliche und psychologische Psychotherapeuten mit neuropsychologischer Zusatzqualifikation erfolgen (Bundesministerium für Gesundheit 2012). Aus Gründen der Qualitätssicherung kann die somatische Abklärung und weiterführende Diagnostik sowie Therapie nicht durch dieselbe Person, sondern muss durch unterschiedliche Behandler erfolgen (Bundesministerium für Gesundheit 2012).

Grundlagen neuropsychologischer Verfahren sind die Restitution (Wiederherstellung), die Kompensation (Ausgleich) sowie Akzeptanz und Adaptation unter Verwendung integrierter Verfahren (z.B. bestimmte psychotherapeutische Verfahren) (Gauggel 2003).

Die Restitution beschreibt die Wiederherstellung oder Verbesserung eingeschränkter oder gestörter Funktionalität von kognitiven oder emotionalen Bereichen aufgrund von Läsionen des zentralen Nervensystems. (Gauggel 2003). Insbesondere zu Beginn einer MS-Erkrankung scheint die Wahrscheinlichkeit besonders groß, durch gezielte Stimulationen neuronaler Netzwerke die Funktionalität wiederherzustellen, sie zu erhalten oder zu verbessern (Penner et al. 2006).

Kompensation beschreibt das Erlernen und Anwenden von internen und externen Strategien mit dem Ziel, dass gesunde und funktionsfähige Areale die eingeschränkten Hirnleistungen ausgleichen. Kompensationsstrategien spielen bei der Behandlung von Patienten mit MS eine zentrale Rolle (DGN 2014). Eine wichtige Bedeutung hat dabei 
- Tab. 4.8 Indikationen (somatische Abklärung) zur neuropsychologischen Therapie (ICD-10-GM)

\begin{tabular}{|c|c|c|}
\hline ICD-10-Code & Titel & Beschreibung nach DIMDI \\
\hline F04 & $\begin{array}{l}\text { Organisches amnestisches Syndrom, nicht durch } \\
\text { Alkohol oder andere psychotrope Substanzen } \\
\text { bedingt }\end{array}$ & $\begin{array}{l}\text { Beeinträchtigungen des Kurz- und Langzeitge- } \\
\text { dächtnisses. Eingeschränkte Fähigkeit, neues } \\
\text { Material zu erlernen. Zeitliche Desorientierung. } \\
\text { Prognose ist abhängig vom Verlauf der zugrunde } \\
\text { liegenden Läsion. }\end{array}$ \\
\hline Aus F06* & \multicolumn{2}{|c|}{$\begin{array}{l}\text { Andere psychische Störungen aufgrund einer Schädigung oder Funktionsstörung des Gehirns oder } \\
\text { einer körperlichen Krankheit }\end{array}$} \\
\hline F06.6 & $\begin{array}{l}\text { Organische emotional labile [asthenische] } \\
\text { Störung }\end{array}$ & $\begin{array}{l}\text { Affektdurchlässigkeit oder -labilität. Ermüdbar- } \\
\text { keit. Vielzahl körperlicher Missempfindungen } \\
\text { (z.B. Schwindel) und Schmerzen als Folge einer } \\
\text { organischen Störung }\end{array}$ \\
\hline F06.7 & Leichte kognitive Störung & $\begin{array}{l}\text { Gedächtnisstörungen, Lernschwierigkeiten, } \\
\text { Konzentrationsschwäche. Geistige Ermüdung. } \\
\text { Objektiv erfolgreiches Lernen wird subjektiv als } \\
\text { schwierig empfunden }\end{array}$ \\
\hline F06.8 & $\begin{array}{l}\text { Sonstige näher bezeichnete organische psy- } \\
\text { chische Störungen aufgrund einer Schädigung } \\
\text { oder Funktionsstörung des Gehirns oder einer } \\
\text { körperlichen Krankheit }\end{array}$ & \\
\hline F06.9 & $\begin{array}{l}\text { Nicht näher bezeichnete organische psychische } \\
\text { Störung aufgrund einer Schädigung oder Funk- } \\
\text { tionsstörung des Gehirns oder einer körper- } \\
\text { lichen Krankheit }\end{array}$ & \\
\hline F07 & $\begin{array}{l}\text { Persönlichkeits- und Verhaltensstörung aufgrund } \\
\text { einer Krankheit, Schädigung oder Funktionsstö- } \\
\text { rung des Gehirns }\end{array}$ & $\begin{array}{l}\text { Persönlichkeits- oder Verhaltensveränderungen } \\
\text { als Rest- oder Begleiterscheinung einer Krank- } \\
\text { heit, Schädigung oder Funktionsstörung des } \\
\text { Gehirns. }\end{array}$ \\
\hline
\end{tabular}

auch das Erkennen und Nutzen externer Hilfestellungen und Unterstützersysteme durch Familien, im beruflichen Umfeld oder durch technische Hilfsmittel und Kommunikationsmittel (Haase et al. 2012). Durch Kompensationsstrategien können zum einen Fertigkeiten wieder erlernt und zum anderen einer physischen wie psychischen dauerhaften Überforderung entgegengewirkt werden. Der sorgfältige Umgang mit den eigenen physischen wie psychischen Ressourcen hat positive Effekte auf das Aktivitätsniveau, die Teilhabe sowie das individuelle Erleben der eigenen Situation.

Um eine Restitution oder Kompensation bestehender Einschränkungen zu erreichen, werden im
Rahmen der neuropsychologischen Rehabilitation integrierte Verfahren eingesetzt. Integrierte Verfahren sind Psychotherapieverfahren, die darauf abzielen, den Patienten in der Krankheitsbewältigung (Adaptation) und Krankheitseinsicht (Akzeptanz) zu unterstützen. Aus Sicht der Betroffenen ist der Erhalt der biografischen Kontinuität (familiär, beruflich) ein zentraler Versorgungsbedarf (Galushko et al. 2014). Familientherapeutische Verfahren helfen, Verständnis für den Betroffenen zu generieren, externe Ressourcen zu identifizieren und das soziale Umfeld in den Prozess der Krankheitsbewältigung einzubeziehen (Gauggel 2003; Patejdl et al. 2015). 
Eine 2014 erschienene systematische Übersichtsarbeit (Meta-Analyse) untersuchte die Wirksamkeit der neuropsychologischen Rehabilitation auf die kognitive Leistungsfähigkeit von Patienten mit MS. Ausgewertet wurden insgesamt 20 Studien. Die meisten der beobachteten Patienten $(\mathrm{n}=966)$ hatten eine RRMS. Der mittlere Einschränkungsgrad lag bei einem EDSS von 3,2 und die mittlere Erkrankungsdauer betrug 14 Jahre. Das Fazit der Meta-Analyse war, dass sich durch gezieltes $\mathrm{Ge}$ dächtnistraining die Gedächtnisleistung und in Kombination mit anderen neurorehabilitativen Interventionen das verbale Erinnerungsvermögen verbessern ließ. Der Evidenzgrad neurorehabilitativer Maßnahmen hinsichtlich einer Reduktion kognitiver Symptome wurde insgesamt jedoch als gering eingestuft. Des Weiteren konnte mittels der Meta-Analyse kein Effekt der untersuchten Maßnahmen auf das seelische Wohlbefinden der $\mathrm{Pa}$ tienten nachgewiesen werden (Rosti-Otajarvi u. Hamalainen 2014). Bei Betrachtung der einzelnen Studien zeigte sich jedoch, dass in $90 \%$ der Fälle (18 von 20 Studien) die jeweiligen Interventionen einen positiven Effekt auf die meisten der untersuchten Outcome-Parameter hatten. Die eher schwache Evidenzlage, die sich aus der Meta-Analyse ergibt, liegt also weniger an den Interventionen per se, sondern ist vielmehr methodisch in der schwierigen Vergleichbarkeit der einzelnen Interventionen begründet (Rosti-Otajarvi u. Hamalainen 2014).

In der Behandlung der MS-assoziierten Fatigue haben sich des Weiteren kognitive Verhaltenstherapie, Programme zum Management der Fatigue unter Berücksichtigung des individuellen Beschwerdebilds und vorhandener Ressourcen sowie das Achtsamkeitstraining, das sich auch auf die eine depressive Symptomatik positiv auswirken kann, bewährt (Patejdl et al. 2015).

Vor dem Hintergrund fehlender standardisierter Verfahren wurde jüngst eine standardisierte Intervention vorgestellt (Metacognitive Training in MS, MaTiMS), die die relevanten neuropsychologischen Komponenten wie Aufmerksamkeit, Gedächtnis, Fatigue, Stress, Depression und Einfühlung berücksichtigt (Pottgen et al. 2015). Die Ergebnisse der Pilotstudie wiesen auf einen positiven Effekt der Intervention hinsichtlich Selbstwirksamkeit,
Verbesserung der Fatigue und Lebensqualität der Teilnehmer hin (Pottgen et al. 2015).

Die neuropsychologischen Verfahren können zusätzlich dazu beitragen, die Adhärenz ( $\downarrow$ Abschn. 4.1.3) und damit den Therapieerfolg der verlaufsmodifizierenden Therapie zu erhöhen. Während die Bedeutung der Adhärenz für die Arzneimitteltherapie in der Behandlung der MS und anderen chronischen Erkrankungen gut untersucht ist, besteht nur wenig Evidenz bezüglich der Auswirkung auf die Therapieadhärenz und den Therapieerfolg bei nicht-medikamentösen rehabilitativen Maßnahmen (Heesen et al. 2014).

\subsubsection{Versorgungssituation}

\section{Studienlage}

Flachenecker et al. (2008) untersuchten die symptombezogene Inanspruchnahme symptomatischer Therapieformen anhand des DMSG-Datensatzes in Deutschland und stellten eine deutliche Unterversorgung hinsichtlich der Inanspruchnahme fest. Die Auswertung spiegelt sehr gut die Versorgungssituation der im Datensatz erfassten Patienten und Einrichtungen wider. Inwieweit sich diese Ergebnisse aber auf die Gesamtversorgungssituation in Deutschland übertragen lassen, ist nicht geklärt. Im Vergleich zu Daten der GKV z.B. sind Patienten mit einer SPMS oder PPMS deutlich häufiger enthalten (Datensatzbeschreibung DMSG > Kap. 4.1.3). Der Zeitpunkt der Datenerhebung liegt außerdem zehn Jahre zurück, entsprechend sind aktuelle Entwicklungen von z.B. neuen Therapieoptionen oder Veränderungen der Versorgungsstrukturen in der Erhebung nicht abgebildet. Des Weiteren fehlen Angaben zum Schweregrad der Beschwerden sowie zum zeitlichen Bezug zwischen Auftreten der Beschwerden und der Initiierung einer möglicher Behandlung (Flachenecker et al. 2008).

Andere Studien setzten sich mit der Qualität der Versorgung auseinander. Beispielsweise Henze et al. (2013) untersuchten in einer bundesweiten Studie die Bedeutung und Behandlung der MSinduzierten Spastik aus Perspektive von Ärzten und Patienten im Jahr 2011 (MObilitätsVErbesserung bei Spastik in Multipler Sklerose, MOVE1-Studie). 
Insgesamt sind 2015 aber nur wenige Studien verfügbar, die die Inanspruchnahme und Qualität der Versorgung in Deutschland unter den aktuellen Rahmen- und Alltagsbedingungen abbilden.

Für die in Frage kommende Arzneimitteltherapie ist in den meisten Fällen die Evidenzlage, die die Grundlage der Leitlinienempfehlungen bildet, nicht sehr belastbar. Der Großteil der Medikamente wird seit vielen Jahren bis Jahrzehnten eingesetzt (• Tab. 4.7) (DGN 2014).

Aufgrund der Vielfältigkeit der Symptome und der Variationsbreite der individuellen Beschwerdebilder, die im Krankheitsbild »MS« zusammengefasst werden, ist es prinzipiell schwierig, einen Therapiestandard zu identifizieren und einer Versorgungsrealität gegenüberzustellen. Die in diesem Kapitel beschriebenen Symptome bilden nicht die Gesamtheit der klinischen Symptomatik ab, sondern stellen lediglich eine Auswahl der häufigsten MS-assoziierten Symptome dar. Der Fokus wird dabei auch auf Faktoren gelegt, die mittlerweile zunehmend zur Beurteilung der Krankheitsaktivität herangezogen werden. Hierzu gehören insbesondere neuropsychologische Beeinträchtigungen, wie kognitive Dysfunktion, Fatigue, Depression und Angst (Stangel et al. 2015).

\section{Allgemeine Inanspruchnahme und Angebotskapazitäten}

\section{- Stationäre (multimodale) Rehabilitation}

Der Zugang zu einer stationären Rehabilitationsmaßnahme erfolgt in der Regel über einen vom Arzt gestellten Antrag bei der Krankenkasse. Bisher war die Beantragung einer stationären Rehabilitation mit vergleichsweise hohen Hürden verbunden. Zum einen ging die Beantragung mit einem hohen bürokratischen Aufwand einher, zum anderen waren nur Ärzte antragsberechtigt, die im Besitz einer bestimmten Zusatzqualifikation waren. Mit der Neufassung der Rehabilitations-Richtlinie des G-BA, welche zum 01. April 2016 in Kraft tritt, dürfen nun alle Vertragsärzte eine Rehabilitationsmaßnahme verordnen. Zudem ist eine deutliche Reduzierung des bürokratischen Aufwands für die Ärzte vorgesehen, sodass Patienten mit MS im Idealfall ein besserer Zugang zu einer stationären Rehabilitation gewährleistet werden kann (Gemeinsamer Bundesausschuss 2015).
$\mathrm{Zu}$ den relevanten leistungserbringenden Einrichtungen zählen stationäre Rehabilitationskliniken ebenso wie ambulante rehabilitative Anbieter. Im Jahr 2013 waren rund $13 \%$ der 1.187 aufgestellten Betten der stationären Vorsorge- und Rehabilitationseinrichtungen in Deutschland Betten der Fachabteilung Neurologie (Statistisches Bundesamt 2013). Je nach Klinik sind insbesondere Neurologen und Psychologen bzw. Psychotherapeuten, Neuropsychologen sowie Ergo- und Physiotherapeuten, Pflegekräfte und Sozialpädagogen in die MS-Rehabilitation involviert.

In einer bundesweiten Umfrage wurde die Behandlungs- und Betreuungsstruktur neurologischer Rehabilitationseinrichtungen mit und ohne MSSchwerpunkt erfasst. Insgesamt konnten 90 Kliniken ( $49 \%$ aller Einrichtungen in 2007) in die Auswertung einbezogen werden (Gesamt $\mathrm{n}=183$, Rücklauf $\mathrm{n}=118$, nicht auswertbare Bögen $=28$ ). Der überwiegende Anteil der Einrichtungen ( $\mathrm{N}=70$; $78 \%$ gab an, keinen MS-Schwerpunkt zu besitzen (allgemein-neurologische Kliniken), während 20 Einrichtungen (22\%) einen expliziten MS-Fokus aufwiesen (MS-Kliniken). Die allgemein-neurologischen Kliniken behandelten vorwiegend Patienten zwischen 25 und 30 Jahren sowie zwischen 60 und 80 Jahren. Die Patienten der MS-Schwerpunktkliniken waren größtenteils zwischen 50 und 70 Jahre alt. Der Behandlungsschwerpunkt aller Reha-Kliniken lag in der weiterführenden Reha und der Anschlussheilbehandlung (•Tab. 4.9). Im Gegensatz zu allgemein-neurologischen Klinken führten Schwerpunktklinken keine Nachsorge und keine berufliche Rehabilitationen durch (Heesen et al. 2010).

Der überwiegende Teil der allgemein-neurologischen und der Schwerpunktkliniken führte sowohl ambulante als auch stationäre Behandlungen durch. Die Betreuungsstruktur als Verhältnis zwischen Patienten- und Therapeutenanzahl zeigte keine wesentlichen Unterschiede zwischen den beiden Einrichtungsarten. In allen Kliniken gehören Physiotherapeuten zur Hauptberufsgruppe. Am wenigsten vertreten sind Sozialpädagogen sowie Musik- und Kunsttherapeuten. Die Anzahl der Pflegekräfte wurde nicht erfasst (Heesen et al. 2010).

In Reha-Einrichtungen mit MS-Schwerpunkt lag die durchschnittliche Behandlungsdauer bei 4,6 
- Tab. 4.9 Schwerpunktsetzung der Reha-Kliniken bezüglich unterschiedlicher Rehabilitationsphasen

\begin{tabular}{|l|l|l|}
\hline Reha-Phase & $\begin{array}{l}\text { MS-Schwerpunktklinik: } \\
\text { Patienten n (\% aller Patienten der } \\
\text { Einrichtungsart) }\end{array}$ & $\begin{array}{l}\text { Allgemein-neurologische Klinik: } \\
\text { Patienten n (\% aller Patienten der } \\
\text { Einrichtungsart) }\end{array}$ \\
\hline $\begin{array}{l}\text { Frührehabilitation } \\
\text { weiterführende Reha }\end{array}$ & $\begin{array}{l}\text { 14 (70\%) } \\
\text { Anschlussheilbehandlung }\end{array}$ & $62(90 \%)$ \\
\hline $\begin{array}{l}\text { Nachsorge und berufliche Reha } \\
\text { aktivierende Pflege }\end{array}$ & $0(0 \%)$ & $67(96 \%)$ \\
\hline $\begin{array}{l}\text { sonstige } \\
\text { Quelle: IGES - Heesen et al. (2010) }\end{array}$ & $2(10 \%)$ & $15(21 \%)$ \\
\hline \begin{tabular}{l} 
Anmerkung: Unter »sonstige« werden v. a. Nachbefragungen subsumiert. \\
\hline
\end{tabular} & $5(25 \%)$ & $15(21 \%)$ \\
\hline
\end{tabular}

Wochen im Vergleich zu 5,4 Wochen in allgemeinneurologischen Kliniken. Insgesamt liegt die durchschnittliche Dauer der stationären neurologischen Rehabilitation über der in anderen Fachbereichen (ca. 3 Wochen). Über $90 \%$ der Schwerpunktkliniken gaben an, ein MS-spezifisches Behandlungsprogramm durchzuführen. Bezüglich der therapeutischen Teilbereiche standen bei fast allen RehaEinrichtungen physiotherapeutische Maßnahmen (v. a. Bobath-Techniken) und Neurosport im Vordergrund. Zum Management von Ataxie und Tremor wurden differenzierte Behandlungen (z. B. Kühlwesten, Eiswasserbäder, Tiefenhirnstimulation in Kooperation) wesentlich häufiger in MS-Schwerpunktkliniken durchgeführt im Vergleich zu allgemeinen Reha-Einrichtungen. Bei etwa $66 \%$ der Patienten mit MS fand eine neuropsychologische Untersuchung statt. Kognitive Trainings sowie Schulungen wurden von allen Rehabilitationskliniken angeboten (Heesen et al. 2010).

In den betrachteten Einrichtungen wurden hauptsächlich sozialrechtliche und Berufsberatungen durchgeführt. Angehörigenberatungen wurden von den allgemein-neurologischen Kliniken häufiger angeboten als von den Schwerpunktkliniken. Individuelle Therapieziele wurden laut Eigenangaben in fast allen Einrichtungen zwischen Arzt und Patient zu Beginn der Therapie festgelegt, allerdings in den wenigsten Fällen schriftlich fixiert. In weniger als $20 \%$ der befragten Kliniken fand eine Evalu- ation mit standardisierten MS-spezifischen Instrumenten z. B. zur Erhebung der Lebensqualität oder zur Bestimmung des EDSS-Wertes statt (Heesen et al. 2010).

In seinem Gutachten wies der Sachverständigenrat zur Begutachtung der Entwicklung im Gesundheitswesen darauf hin, dass der Bedarf an medizinischer Rehabilitation das vorhandene Angebot deutlich übersteigt (Sachverständigenrat zur Begutachtung der Entwicklung im Gesundheitswesen 2014). Auch in Zukunft ist aufgrund der Alterung der Bevölkerung und der (absoluten) Zunahme chronischer Erkrankungen wie auch der MS mit einem steigenden ungedeckten Versorgungsbedarf zu rechnen.

\section{- Physiotherapie (Heilmittel)}

Die ambulante Physiotherapie zählt zu den Heilmitteln und muss ambulant vertragsärztlich verordnet werden. Ein direkter Zugang ist (noch) nicht möglich. Der Zugang zur Physiotherapie hat sich in den letzten Jahren für Patienten mit MS verbessert. Bis vor einigen Jahren bestanden vergleichsweise hohe Hürden, was eine dauerhafte Verordnung der Physiotherapie anging. In einem Merkblatt in Zusammenhang mit der Heilmittelrichtlinie regelte der Gemeinsame Bundesausschuss (G-BA) in 2013, dass Patienten mit dauerhaftem Heilmittelbedarf entsprechende dauerhafte Anwendungen durch ihre Krankenkasse genehmigt bekommen können. 
Die MS wird dabei in einer Liste von Erkrankungen geführt, der ein dauerhafter Heilmittelbedarf bescheinigt wird (siehe Kapitel 6.5.4) (Gemeinsamer Bundesausschuss 2011).

Die Anzahl an beschäftigten Physiotherapeuten und zugelassenen Physiotherapie-Praxen steigt ( Kap. 6.5.4). Dennoch gilt die Physiotherapie als Engpassberuf, das heißt, es gibt mehr unbesetzte Stellen als Arbeitsuchende. Der Bedarf wird im Zuge des demographischen Wandels grundsätzlich weiter steigen, die Ausbildungszahlen sind jedoch rückläufig (Grosch 2015).

Informationen zur Inanspruchnahme von ambulanten Rehabilitationsmaßnahmen bei Patienten mit MS sind kaum vorhanden.

\section{- Neuropsychologische Therapie}

Der Zugang zu neuropsychologischen Therapieleistungen erfolgt für Patienten mit MS in der Regel durch die Überweisung zu einem Neuropsychologen. Die Gesellschaft für Neuropsychologie (GNP) (2015) verzeichnet bundesweit aktuell (Stand: 18.09.2015) 287 ambulant und stationär tätige zertifizierte Neuropsychologen.

Neuropsychologen sind sowohl im stationären Bereich als auch in der ambulanten Versorgung tätig. Gemäß einer bundesweiten Totalerhebung zur Versorgungssituation im Bereich der ambulanten neuropsychologischen Therapie aus dem Jahr 2009 wurden 181 ambulant tätige zertifizierte Neuropsychologen berichtet (insgesamt etwa 200 ambulant tätige Neuropsychologen bundesweit in 2009) (Mühlig et al. 2009). Basierend auf diesen Angaben errechneten die Autoren eine Versorgungsdichte von einem Neuropsychologen pro 356.874 Einwohner im Jahr 2009, wobei der Großteil der ambulant tätigen Neuropsychologen in den westlichen Bundesländern praktizierte (Verhältnis 3:1) (Mühlig et al. 2009).

Flachenecker et al. schlussfolgerten in einer ihrer Auswertungen des DMSG-Datensatzes, dass auch aufgrund der geringen Angebotskapazitäten für die neuropsychologische Versorgung von einer erheblichen Unterversorgung von Patienten mit MS auszugehen ist (Flachenecker et al. 2008).

Neben der Verfügbarkeit spielt die barrierefreie Zugänglichkeit existierender Versorgungsangebote eine wichtige Rolle für Patienten mit MS. Die wohnortnahe Versorgung ist ein wichtiger Versorgungs- bedarf, um trotz krankheitsbedingter Einschränkungen und Behinderungen Behandlungsprogramme in Anspruch nehmen zu können (Galushko et al. 2014). Bei über der Hälfte der neuropsychologischen Einrichtungen reisten die Patienten aber aus einem Umkreis von 11 bis $50 \mathrm{~km}$ für die Inanspruchnahme einer neuropsychologischen Therapie an, bei $18 \%$ der Einrichtungen betrug das Einzugsgebiet über $50 \mathrm{~km}$. Bei einem Drittel der ambulanten Einrichtungen betrug das Einzugsgebiet unter $10 \mathrm{~km}$ (Mühlig et al. 2009).

Unter Berücksichtigung des jährlichen Neuerkrankungsrisikos (Inzidenz) von rund 550.000 Hirnschädigungen insgesamt in der Bevölkerung (davon schätzungsweise $10 \%$ mit Indikation für eine neuropsychologische Therapie) (Kasten et al. 1997), der steigenden Inzidenz und Prävalenz der MS-Erkrankungen und den Angebotsstrukturen (287 ambulant tätige Neuropsychologen) ist auch aktuell von einem erheblichen ungedeckten Bedarf in der ambulanten neuropsychologischen Versorgung auszugehen.

\section{- Arzneimittel}

Die Leitlinien zur Diagnose und Therapie der Multiplen Sklerose sehen eine Behandlung mit Arzneimitteln im Rahmen der symptomatischen Therapie bei verschiedenen Indikationen vor (DGN 2014). Innerhalb der GKV-Population Bayerns erhielten 2009 40,6 \% der Patienten mit MS mindestens eine Verordnung eines Medikaments für die symptomatische Therapie. $33 \%$ der Patienten erhielten mindestens eine Verordnung aus der Gruppe der nichtsteroidalen Antirheumatika NSAR. $12 \%$ der Patienten erhielten Hypnotika und Sedativa, $7 \%$ Opioide und bei $4 \%$ war mindestens eine Verordnung für ein Medikament aus der Gruppe der Neuroleptika dokumentiert (Höer et al. 2014).

Das am häufigsten in Anspruch genommen Medikament war Baclofen, welches zur Behandlung der Spastik eingesetzt wird. Auch Tolperison und Gabapentin dienen der Behandlung von Spastiken. Bei Citalopram und Mirtazapin handelt es sich um Antidepressiva, welche auch in der Schmerztherapie eingesetzt werden (• Abb. 4.9) (Höer et al. 2014).

Über $50 \%$ der Arzneimittelverordnungen für die symptomatische Therapie wurden von Neurologen und Nervenärzten ausgestellt. Gut ein Drittel 


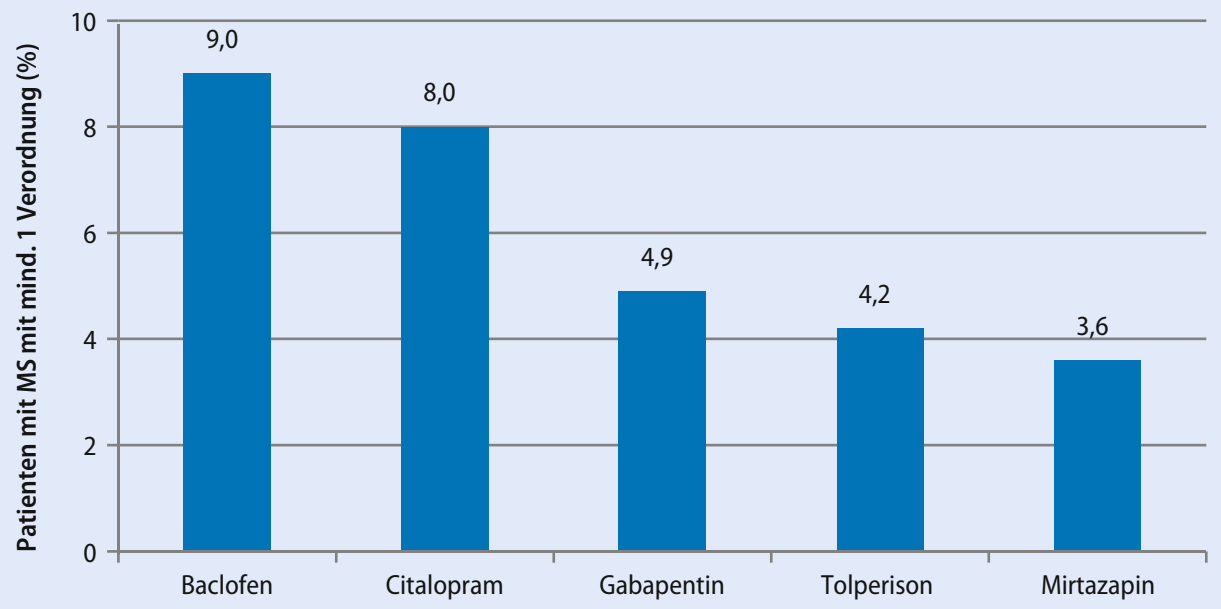

- Abb. 4.9 Anteil an Patienten mit mindestens einer arzneimittelspezifischen Verordnung für die symptomatische Therapie der MS (2009) Quelle: IGES - Höer et al. (2014)

der Verordnungen für NSAR erfolgte durch Hausärzte (Höer et al. 2014).

\section{Symptombezogene Inanspruchnahme}

- Neuropsychologische Symptome (Kognitive Beeinträchtigungen, Fatigue und Depression) Symptome des neuropsychologischen Formenkreises wie kognitive Beeinträchtigungen, Fatigue, Depression und Angst zählen zu den häufigsten Krankheitszeichen der MS (Beschreibung der Symptome siehe auch $>$ Kap. 1.2). Die Symptome sind bei Patienten mit MS häufig eng mit einander assoziiert (Nagaraj et al. 2013).

Die Therapie der kognitiven Beeinträchtigung kann durch teilleistungsspezifisches kognitives Training, durch die Vermittlung von Kompensationsstrategien und einer begleitenden Psychotherapie, welche auch die Angehörigen berücksichtigt, erfolgen. Studien zeigen, dass spezifisches Aufmerksamkeitstraining über 4-12 Wochen eine Verbesserung der Informationsverarbeitungsgeschwindigkeit und des Gedächtnisses für 6-12 Monate nach sich ziehen kann (Brenk et al. 2008, O'Brien et al. 2008). Zudem deuten Studien darauf hin, dass sich ein gezieltes Arbeitsgedächtnistraining darüber hinaus positiv auf die Fatiguesymptomatik auswirken kann (Vogt et al. 2008; Vogt et al. 2009).
Zur medikamentösen Behandlung liegen derzeit keine Empfehlungen vor (DGN 2014). Analysen des DMSG-Datensatzes zeigten, dass $83 \%$ der Patienten mit MS, die eine kognitive Beeinträchtigung aufwiesen, unbehandelt blieben. Etwa $8 \%$ erhielten eine medikamentöse oder nicht-medikamentöse Therapie (Flachenecker et al. 2008).

Die Therapie der Fatigue beginnt mit der Behandlung der zugrunde liegenden Primärerkrankung. Dazu zählt zum einem die frühzeitige an die Krankheitsaktivität angepasste verlaufsmodifizierende Therapie, die über die Reduktion der immunologischen Korrelate der MS, die eventuell damit zusammenhängende Fatigue-Symptomatik verringert ( $\triangleright$ Abschn. 4.1.1). In der Behandlung der Fatigue kommen weitere nicht-medikamentöse und medikamentöse Ansätze (• Tab. 4.7) inklusive der Therapie auslösender Ursachen (wie gestörter Nachtschlaf bei Blasenstörungen, Unterfunktion der Schilddrüse) in Frage (Pateijdl 2015). Bewegung und sportliches Training können beispielsweise wirksam zu einer Linderung der FatigueSymptomatik beitragen. Andreasen et al. kommen in einer Übersichtsarbeit zu dem Ergebnis, dass sich körperliche Aktivität in Form von verschiedenen Modellen der kontinuierlichen Bewegungstherapie insgesamt günstig auf die Fatigue-Symptoma- 
tik auswirkt (Andreasen et al. 2011). In einer 2015 erschienenen Untersuchung zeigten Kerling et al., dass eine Kurzintervention (zwei Trainingseinheiten pro Woche von 40 Minuten Dauer, moderate Intensität, über drei Monate) bestehend aus Ausdauertraining oder als Kombination von Ausdauerund Widerstandstraining zu einer signifikanten Verbesserung der Fatigue-Symptomatik und der Lebensqualität von Patienten mit MS führte. Die Form des Trainings (Ausdauertraining allein oder in Kombination mit Widerstandstraining) spielte dabei keine Rolle (Kerling et al. 2015). Aufgrund der multifaktoriellen Ätiologie der Fatigue ist eine Kombination verschiedener Verfahren häufig am vielversprechendsten (DGN 2014; Patejdl et al. 2015).

Auf Basis von Daten des DMSG-Datensatzes konnte gezeigt werden, dass bei $79 \%$ der Patienten mit MS, die an einer Fatigue litten, keine Behandlung dokumentiert war. Etwa $14 \%$ der Betroffenen erhielten eine medikamentöse und $7 \%$ eine nichtmedikamentöse Therapie. Eine Kombination der Verfahren wurde bei keinem der Patienten dokumentiert (Flachenecker et al. 2008).

Für die Therapie der Depression kommen psychotherapeutische, medikamentöse oder somatische Verfahren in Betracht (DGPPN et al. 2009). Analysen des DMSG-Datensatzes zeigten hier, dass bei $63 \%$ der Patienten mit MS die vorliegende Depression behandelt wurde (bei $56 \%$ medikamentös und bei $5 \%$ der Betroffenen durch nicht medikamentöse Verfahren, bei $2 \%$ in Kombination) (Flachenecker et al. 2008).

- Motorische Symptome und Einschränkungen der Mobilität (am Beispiel der Spastik und Ataxie)

Die Therapie der Wahl bei motorischen Symptomen und Einschränkungen der Mobilität ist die Physiotherapie (DGN 2014). Eine medikamentöse Behandlung von Spastik wird erst empfohlen, wenn mit Hilfe der Physiotherapie keine ausreichenden Erfolge verzeichnet werden konnten. Verwendet werden beispielsweise orale Antispastika wie Baclofen, Tolperison oder Tizanidin (•Tab. 4.7). Invasive Therapiemaßnahmen mit beispielsweise Botulinum-Toxin A werden bei ausgeprägter lokaler Spastik verwendet (DGN 2014).
Analysen des DMSG-Datensatzes hinsichtlich der Therapie motorischer Symptome und Einschränkungen ergaben, dass etwa $31 \%$ der Patienten mit MS eine unbehandelte Spastik aufwiesen. Bei 18 \% der Patienten mit spastischen Symptomen war eine nicht-medikamentöse, bei $38 \%$ eine medikamentöse und bei $13 \%$ eine Kombinationstherapie dokumentiert. Die Ataxie blieb in $62 \%$ der Fälle unbehandelt, $29 \%$ erhielten eine nicht-medikamentöse und $8 \%$ eine medikamentöse Therapie (Flachenecker et al. 2008).

Eine Querschnittsuntersuchung zur Bedeutung und Behandlung der Spastik bei MS (MOVE-1Studie) kam zu dem Ergebnis, dass sich durch die zum Studienzeitpunkt verfügbaren Medikamente aus Perspektive der Patienten und Ärzte keine ausreichende Versorgung der Spastik und ihren assoziierten Symptomen herstellen ließ. Die Studie berücksichtigte dabei Daten von 414 Patienten aus 42 verschiedenen Behandlungseinrichtungen in Deutschland. Insbesondere Nebenwirkungen und unzureichende Wirksamkeit der verfügbaren Antispastika trugen aus Sicht der Ärzte und Patienten zur schlechten Versorgungssituation bei. Ein Drittel der Patienten suchte zusätzliche Hilfe durch Selbstmedikation. Die Autoren sehen aber Potenzial in einer Ausweitung und Optimierung physiotherapeutischer Maßnahmen und in der Verwendung von Cannabinoiden (Henze et al. 2013).

\section{- Schmerz}

Für die Behandlung neuropathischer Schmerzen kommen unter anderem Antidepressiva und Opioide zum Einsatz, da diese den Anstieg der den Schmerz inhibierenden Neurotransmitter anregen bzw. den der Schmerz verursachenden hemmen (DMSG 2004; Wiendl et al. 2010). Neben den neuropathischen Schmerzen können Schmerzen im Zusammenhang mit Spastiken, muskulären Fehlstellungen oder anderen Symptomen der MS (indirekte Schmerz) oder in Zusammenhang mit der Arzneimitteltherapie (arzneimittelassoziierter Schmerz) auftreten. In der Behandlung der Schmerzen werden zusätzlich physikalische Therapien, Massagen oder gezielte Bewegungen eingesetzt, um beispielsweise die mit der Spastik oder mit Fehlstellungen assoziierten Schmerzen $\mathrm{zu}$ behandeln (DMSG 2004). 
Bei $44 \%$ der Patienten des DMSG-Datensatzes war keine Behandlung vorhandener Schmerzen dokumentiert. 47 \% der Patienten mit Schmerzsymptomatik wurden medikamentös, $7 \%$ nicht-medikamentös und $2 \%$ in Kombination therapiert (Flachenecker et al. 2008).

\section{- Störungen der Blasenfunktion und Sexualität}

Grundlage der Behandlung von Blasenstörungen ist die Verhaltenstherapie. Diese beinhaltet die Bestimmung der patientenindividuellen richtigen Trinkmenge, das Führen von Miktionstagebüchern, um den Entleerungsrhythmus entsprechend anpassen zu können, sowie Beckenbodentraining. Weiterhin wird der intermittierende Selbstkatheterismus empfohlen (DGN 2014).

Die medikamentöse Standardtherapie stellen Anticholinergika wie beispielsweise Oxybutynin und Tolterodin dar (•Tab. 4.7). Deren Wirksamkeit sowie ein positiver Einfluss auf die Lebensqualität gilt als belegt, und sie werden entsprechend auch in den Leitlinien zur Therapie der überaktiven Blase empfohlen (DGGG et al. 2010). Zur Vermeidung von Harnwegsinfekten können zur Harnansäuerung Methionin oder Cranberry-Präparate verwendet werden. Desmopressin, welches als nasales Spray verabreicht wird, findet bei erheblicher Pollakisurie Anwendung. Eine Behandlung der überaktiven Blase bei MS mit Botulinum-Toxin ist derzeit aufgrund mangelnder Langzeitstudien nicht zugelassen, gilt jedoch als vielversprechend (DGN 2014).

Als invasive Therapiemaßnahmen zur Reduktion der Blasenaktivität kommen die sakrale Neuromodulation sowie rekonstruktive operative Verfahren zum Einsatz. Für letztere Ansätze liegen jedoch keine Langzeiterfahrungen bei Patienten mit MS vor und sie sollten daher nur als Ultima ratio erfolgen (DGN 2014).

Nach Flachenecker et al. (2008) bleiben $45 \%$ der Blasenstörungen bei MS Patienten des DMSGDatensatzes unbehandelt. Lediglich $36 \%$ der Patienten erhalten eine medikamentöse Therapie. $14 \%$ der Patienten bekommen eine nicht-medikamentöse Behandlung und weitere $5 \%$ erhielten beides (Flachenecker et al. 2008).

Auch die Behandlung der sexuellen Dysfunktion ist Teil der symptomatischen Therapie bei Mul- tipler Sklerose. Gemäß der Leitlinie kommen auch bei der sexuellen Dysfunktion neben psychotherapeutischen Verfahren medikamentöse und invasive Verfahren zum Einsatz. Für die medikamentöse Behandlung der erektilen Dysfunktion werden insbesondere Sildenafil, Tadalafil und Vardenafil empfohlen. Weiterhin kann eine intrakavernöse Applikation von Prostaglandinen durch einen Urologen erfolgen. Für Patientinnen mit MS stehen bei mangelnder Lubrifikation der Scheide und Dyspareunie hormonhaltige Cremes zur Verfügung (DGN 2014).

Untersuchungen zeigen, dass sich die betroffenen Patienten hinsichtlich der sexuellen Dysfunktionen unzureichend versorgt fühlen. Beispielsweise gaben $86 \%$ der Patienten an, es bestünde ein Informations- bzw. Aufklärungsbedarf hinsichtlich sexueller Funktionsstörrungen im Rahmen der Erkrankung. Tatsächlich haben nur ein Drittel der Männer und ein Zehntel der Frauen Unterstützung bezüglich ihrer sexuellen Fragen durch Ärzte erfahren. Zudem wünschen sich $46 \%$ der betroffenen Frauen und 36 \% der Männer Paargespräche zu dieser Thematik (Goecker et al. 2006).

\section{Inanspruchnahme Pflege}

Bei Progredienz der Erkrankung nehmen dauerhafte Beschwerden und bleibende Beeinträchtigungen zu. Wenn die individuellen Ressourcen nicht mehr ausreichen, um den Anforderungen des Alltags gerecht zu werden, liegt gemäß $\$ 14$ SGB XI Pflegebedürftigkeit vor ${ }^{1}$. Um Zugang zu Leistungen der Pflegeversicherung zu erhalten, muss bei der Pflegekasse ein Antrag auf Pflegeleistungen gestellt werden. Die Pflegekasse lässt daraufhin durch den Medizinischen Dienst der Krankenversicherung überprüfen, ob die Voraussetzungen der Pflegebedürftigkeit beim Antragsteller erfüllt sind und in welchem Ausmaß diese vorliegt ${ }^{2}$. Aktuell erfolgt die Zuordnung der Pflegebedürftigkeit in eine von drei Pflegestufen (Pflegestufe I: erheblich Pflegebedürftige, Pflegestufe II: Schwerpflegebedürftige, Pflegestufe III: Schwerstpflegebedürftige) ${ }^{3}$. Im Zuge der Neuformulierung des Pflegebedürftigkeitsbegriffs im Pflegestärkungsgesetz II wird die Beeinträchtigung der

\footnotetext{
$1 \S 14$ SGB XI

$2 \S 18$ SGB XI

$3 \S 15 \mathrm{SGB} X \mathrm{I}$
} 
Selbstständigkeit ab 2017 in fünf Pflegegraden erfolgen und kognitive und psychische Beeinträchtigungen dabei stärker berücksichtigen als bisher ( $\triangleright$ Kap. 6.1). Eine Analyse auf Grundlage von Versichertendaten der Barmer GEK zeigte, dass gut $20 \%$ der Patienten mit MS in 2010 gemäß SGB XI pflegebedürftig waren und Pflegeleistungen in Anspruch genommen haben. 8,5\% der Patienten mit MS hatten Pflegestufe I, weitere $8,1 \%$ wurden der Pflegestufe II zugeordnet und $4 \%$ der Patienten Pflegestufe III (weitere 0,2 \% der MS-Patienten wurden als Härtefall der Pflegestufe III eingestuft). Der Anteil der Versicherten mit Pflegestufe III lag damit rund zehnmal höher im Vergleich zu allen Versicherten der Sozialen Pflegeversicherung mit 0,4\%. Die Patienten behielten ihre Pflegestufen dabei konstant über 300 Tage im Jahr. Am häufigsten (12,6 \% der Patienten) wurde das Pflegegeld nach $₫ 37$ SGB XI beansprucht, gefolgt von den Sachleistungen nach $\$ 36$ SGB XI mit 5,6 \% (IGES Institut 2014).

Open Access Dieses Kapitel wird unter der Creative Commons Namensnennung-Nicht kommerziell 4.0 International Lizenz (http://creativecommons.org/licenses/by-nc/4.0/deed.de) veröffentlicht, welche für nicht kommerzielle Zwecke die Nutzung, Vervielfältigung, Bearbeitung, Verbreitung und Wiedergabe in jeglichem Medium und Format erlaubt, sofern Sie den/die ursprünglichen Autor(en) und die Quelle ordnungsgemäß nennen, ein Link zur Creative Commons Lizenz beifügen und angeben, ob Änderungen vorgenommen wurden.

Etwaige Abbildungen oder sonstiges Drittmaterial unterliegen ebenfalls der genannten Creative Commons Lizenz, sofern sich aus der Abbildungslegende oder der Quellreferenz nichts anderes ergibt. Sofern solches Drittmaterial nicht unter der genannten Creative Commons Lizenz steht, ist eine Vervielfältigung, Bearbeitung oder öffentliche Wiedergabe nur mit vorheriger Zustimmung des betreffenden Rechteinhabers oder auf der Grundlage einschlägiger gesetzlicher Erlaubnisvorschriften zulässig.

\section{Literatur}

Andreasen AK, Stenager E, Dalgas U (2011) The effect of exercise therapy on fatigue in multiple sclerosis. Mult Scler 17(9), 1041-1054. DOI: 10.1177/1352458511401120

atd arznei-telegramm Arzneimitteldatenbank (2015) Startseite. Berlin. http://www.arznei-telegramm.de/db/atdstart.php3 [Abruf am: 16. Dezember 2015].

Augurzky B, Reichert A, Scheuer M (2011) Faktenbuch Medizinische Rehabilitation 2011. (Heft 66). Materialien. Essen: Rheinisch-Westfälisches Institut für Wirtschaftsforschung. ISSN: 1312-3573.

Beer S, Khan F, Kesselring J (2012) Rehabilitation interventions in multiple sclerosis: an overview. J Neurol 259(9), 19942008. DOI: 10.1007/s00415-012-6577-4.

Bischoff C, Schreiber H, Bergmann A (2012) Background information on multiple sclerosis patients stopping ongoing immunomodulatory therapy: a multicenter study in a community-based environment. J Neurol Neurophysiol 259(11), 2347-2353. DOI: 10.1007/s00415-012-6499-1.

Brenk A, Laun K, Haase CG (2008) Short-term cognitive training improves mental efficiency and mood in patients with multiple sclerosis. Eur Neurol 60(6), 304-309. DOI: $10.1159 / 000157885$.
Bundesministerium für Gesundheit (2012) Bekanntmachung eines Beschlusses des Gemeinsamen Bundesausschusses über eine Änderung der Richtlinie Methoden vertragsärztliche Versorgung: Neuropsychologische Therapie. Bundesanzeiger (BAnz.). https://www.g-ba.de/downloads/39-261-1415/2011-11-24_MVV-RL_NeuroPsych_ BAnz.pdf [Abruf am: 15. Dezember 2015].

Butler M, Forte ML, Schwehr N, Carpenter A, Kane RL (2015) Decisional Dilemmas in Discontinuing Prolonged Disease-Modifying Treatment for Multiple Sclerosis. Rockville (MD): Agency for Healthcare Research and Quality.

Campbell M, Fitzpatrick R, Haines A, Kinmonth AL, Sandercock P, Spiegelhalter D, Tyrer P (2000) Framework for design and evaluation of complex interventions to improve health. BMJ 321(7262), 694-696.

DGGG, AGUB, DGU, AUB Österreich, AUG Schweiz (2010) Die überaktive Blase (ÜAB). http://www.awmf.org/uploads/ tx_szleitlinien/015-007l_S2k_Ueberaktive_ Blase_2010-abgelaufen.pdf [Abruf am: 16. Dezember 2015].

DGN (Hrsg.) (2012) Multiprofessionelle neurologische Rehabilitation. Entwicklungsstufe: S1, Stand: September 2012, Gültig bis: 31. Juli 2014 (AWMF-Registernummer: 030/122). Deutsche Gesellschaft für Neurologie. http:// www.dgn.org/images/red_leitlinien/LL_2012/pdf/II_87_ 
multiprofessionelle_neurologische_rehabilitation.pdf [Abruf am: 17. Dezember 2015].

DGN (Hrsg.) (2014) Leitlinien für Diagnostik und Therapie in der Neurologie. Diagnose und Therapie der Multiplen Sklerose. Entwicklungsstufe: S2e. Stand: Januar 2012, Ergänzung August 2014. Gültig bis 2017. (AWMF-Registernummer: 030/050). Deutsche Gesellschaft für Neurologie. http://www.awmf.org/uploads/tx_szleitlinien/ 030-050l_S2e_Multiple_Sklerose_Diagnostik_Therapie_ 2014-08_verlaengert.pdf [Abruf am: 04. November. 2015].

DGN (Hrsg.) (2015) Leitlinien für Diagnostik und Therapie in der Neurologie. Pharmakologisch nicht interventionelle Therapie chronisch neuropathischer Schmerzen. Entwicklungsstufe: S1. Veröffentlicht September 2012, Ergänzt 7.1.2014, Gültig bis 31. Dezember 2016. (AWMF-Registernummer: 030/114). Berlin: Deutsche Gesellschaft für Neurologie. http://www.awmf.org/ uploads/tx_szleitlinien/030-114I_S1_Neuropathischer_ Schmerzen_Therapie_2014-01.pdf [Abruf am: 18. Dezember 2015].

DGPPN, BÄK, KBV, AWMF, AkdÄ, BPtK, BApK, DAGSHG, DEGAM, DGPM, DGPs, DGRW (Hrsg. für die Leitliniengruppe Unipolare Depression) (2009) S3-Leitlinie/Nationale VersorgungsLeitlinie Unipolare Depression Kurzfassung, 1. Auflage. Version 5. 2009, zuletzt verändert: Juni 2015. DOI: 10.6101/AZQ/000240. http:// www.awmf.org/uploads/tx_szleitlinien/nvl-005k_Unipolare_Depression-2015-07_verlaengert.pdf [Abruf am: 15. Dezember 2015].

DIMDI (2014) ICD-10-GM Version 2015: Kapitel V Psychische und Verhaltensstörungen (F00-F99): Organische, einschließlich symptomatischer psychischer Störungen (F00-F09). Letzte Aktualisierung: 19. September 2014. Köln. http://www.dimdi.de/static/de/klassi/icd-10-gm/ kodesuche/onlinefassungen/htmlgm2015/block-f00-f09. htm [Abruf am: 15. Dezember 2015].

DMSG (2014) msregister. Hannover: Deutsche Multiple Sklerose Gesellschaft Bundesverband e.V. http://www.dmsg. de/msregister/ [Abruf am: 03. Februar 2016].

DMSG (2004): Symptomatische Therapie der Multiplen Sklerose - Aktuelle Therapieempfehlungen. Multiple Sklerose Therapie Konsensus Gruppe (MSTKG). Hannover: Deutsche Multiple Sklerose Gesellschaft Bundesverband e.V.

DMSG Berlin e.V. (2015) Rehabilitation. Berlin. http://www. dmsg-berlin.de/multiple-sklerose/rehabilitation.html [Abruf am: 14. Dezember 2015].

Filippini G, Del Giovane C, Vacchi L, D'Amico R, Di Pietrantonj C, Beecher D, Salanti G (2013) Immunomodulators and immunosuppressants for multiple sclerosis: a network meta-analysis. Cochrane Database Syst Rev 6, CD008933. DOI: 10.1002/14651858.CD008933.pub2.

Fisniku LK, Brex PA, Altmann DR, Miszkiel KA, Benton CE, Lanyon R, Thompson AJ, Miller DH (2008a) Disability and T2 MRI lesions: a 20-year follow-up of patients with relapse onset of multiple sclerosis. Brain 131(Pt 3), 808817. DOI: 10.1093/brain/awm329.
Fisniku LK, Chard DT, Jackson JS, Anderson VM, Altmann DR, Miszkiel KA, Thompson AJ, Miller DH (2008b) Gray matter atrophy is related to long-term disability in multiple sclerosis. Ann Neurol 64(3), 247-254. DOI: 10.1002/ ana.21423.

Flachenecker P, Stuke K, Elias W, Freidel M, Haas J, PitschnauMichel D, Schimrigk S, Zettl UK, Rieckmann P (2008) Multiple sclerosis registry in Germany: results of the extension phase 2005/2006. Deutsches Ärzteblatt 105(7), 113-119. DOI: 10.3238/arztebl.2008.0113.

Fricke U, Beck T (2014) Neue Arzneimittel: Fakten und Bewertungen. Bd. 21. Stuttgart: Wissenschaftliche Verlagsgesellschaft mbH. ISBN: 978-3-8047-3182-0.

Gajofatto A, Turatti M, Bianchi MR, Forlivesi S, Gobbin F, Azzara A, Monaco S, Benedetti MD (2015) Benign multiple sclerosis: physical and cognitive impairment follow distinct evolutions. Acta Neurol Scand 133(3), 183-191. DOI: 10.1111/ane.12442.

Galushko M, Golla H, Strupp J, Karbach U, Kaiser C, Ernstmann $\mathrm{N}$, Pfaff H, Ostgathe C, Voltz R (2014) Unmet needs of patients feeling severely affected by multiple sclerosis in Germany: a qualitative study. J Palliat Med 17(3), 274-281. DOI: $10.1089 / j p m .2013 .0497$.

Gauggel S (2003) Grundlagen und Empirie der Neuropsychologischen Therapie: Neuropsychotherapie oder Hirnjogging? Z Neuropsychol 14(4), 217-246. DOI: http:// dx.doi.org/10.1024/1016-264X.14.4.217.

Gemeinsamer Bundesausschuss (2015) Beschluss des Gemeinsamen Bundesausschusses über eine Änderung der Rehabilitations-Richtlinie: Vereinfachung Verordnungsverfahren/Qualifikationsanforderungen. Berlin: Gemeinsamer Bundesausschuss. https://www.g-ba.de/downloads/39-261-2361/2015-10-15_Re-RL_Vereinachung-Verordnungsverf.pdf [Abruf am: 10. Februar 2016].

Gemeinsamer Bundesausschuss (2011) Merkblatt Genehmigung langfristiger Heilmittelbehandlungen nach $\S 32$ Abs. 1a SGB V in Verbindung mit $\S 8$ Abs. 5 HeilmittelRichtlinie. https://www.g-ba.de/downloads/17-98-3382/ 2013-09-19_HeilM-RL_Merkblatt\%20mit\%20Anlage.pdf [Abruf am: 10. Februar 2016].

Gesellschaft für Neuropsychologie (GNP) e.V. (2013) Was ist Neuropsychologie? Letzte Aktualisierung: 24. Juni 2013. Fulda. http://www.gnp.de/_de/fs-Was-ist-Neuropsychologie.php [Abruf am: 15. Dezember 2015].

Gesellschaft für Neuropsychologie (GNP) e.V. (2015) Zertifizierte Neuropsychologen der GNP - Behandlerliste. 22.09.2015: 287 Einträge. 36001 Fulda: Gesellschaft für Neuropsychologie (GNP) e.V. http://www.gnp.de/_de/ fs-Behandlerliste.php [Abruf am: 22. September 2015].

Glaeske G, Schicktanz C (2014) BARMER GEK Arzneimittelreport 2014: Auswertungsergebnisse der BARMER GEK Arzneimitteldaten aus den Jahren 2012 bis 2013. Schriftenreihe zur Gesundheitsanalyse, Bd. 26. Siegburg: Asgard Verlagsservice. ISBN: 978-3-943-74491-0. http:// www.zes.uni-bremen.de/uploads/News/2014/140526_ AMReport_2014_Internet.pdf [Abruf am: 18. August 2014]. 
Goecker D, Rösing D, Beier KM (2006) Der Einfluss neurologischer Erkrankungen auf Partnerschaft und Sexualität: Unter besonderer Berücksichtigung der Multiplen Sklerose und des Morbus Parkinson. Urologe 45(8), 992-998. DOI: 10.1007/s00120-006-1094-7.

Gold R, Hartung HP, Stangel M, Wiendl H, Zipp F (2012) Therapieziele von Basis- und Eskalationstherapien zur Behandlung der schubförmig-remittierenden Multiplen Sklerose. Akt Neurol 39(7), 342-350. DOI: 10.1055/s-0032-1305248.

Golla H, Galushko M, Pfaff H, Voltz R (2012) Unmet needs of severely affected multiple sclerosis patients: the health professionals' view. Palliat Med 26(2), 139-151. DOI: 10.1177/0269216311401465.

Gorenoi V, Schonermark MP, Hagen A (2008) Interventions for enhancing medication compliance/adherence with benefits in treatment outcomes. GMS Health Technol Assess 3, Doc14. ISSN: 1861-8863.

Grosch M (2015) Engpassberuf Physiotherapie. https://physiotherapeuten.de/engpassberuf-physiotherapie/\#.VnKvc nhCig [Abruf am: 17. Dezember 2015].

Haase R, Schultheiss T, Kempcke R, Thomas K, Ziemssen T (2012) Use and acceptance of electronic communication by patients with multiple sclerosis: a multicenter questionnaire study. J Med Internet Res 14(5), e135. DOI: 10.2196/jmir.2133.

Hansen K, Schussel K, Kieble M, Werning J, Schulz M, Friis R, Pohlau D, Schmitz N, Kugler J (2015) Adherence to Disease Modifying Drugs among Patients with Multiple Sclerosis in Germany: A Retrospective Cohort Study. PLoS One 10(7), e0133279. DOI: 10.1371/journal. pone.0133279.

Hapfelmeier A, Dippel FW, Schinzel S, Holz B, Seiffert A, Mäurer M (2013) Aktuelle Aspekte zur Versorgungssituation und zu den Behandlungskosten bei Patienten mit Multipler Sklerose in Deutschland. Gesundh ökon Qual manag 19, 210-216. DOI: 10.1055/s-00331335883.

Häussler B, Höer A, de Millas C (Hrsg.) (2015) ArzneimittelAtlas 2015: Der Arzneimittelverbrauch in der GKV. Berlin: IGES Institut GmbH. ISBN: 978-3-9808407-5-0.

Häussler B, Höer A, Hempel E (Hrsg.) (2014) ArzneimittelAtlas 2014: Der Arzneimittelverbrauch in der GKV. Berlin, Heidelberg: Springer Verlag. ISBN: 978-3-662-43446-8.

Heesen C, Bruce J, Feys P, Sastre-Garriga J, Solari A, Eliasson L, Matthews V, Hausmann B, Ross AP, Asano M, ImonenCharalambous K, Kopke S, Clyne W, Bissell P (2014) Adherence in multiple sclerosis (ADAMS): classification, relevance, and research needs. A meeting report. Mult Scler 20(13), 1795-1798. DOI: 10.1177/1352458514531348.

Heesen C, Berger B, Hamann J, Kasper J (2006) Empowerment, Adhärenz, evidenzbasierte Patienteninformation und partizipative Entscheidungsfindung bei MS -Schlagworte oder Wegweiser? Neurol Rehabil 12(4), 232-238

Heesen C, Stückrath E, Köpke S, Hauptmann B, Henze T (2010) Rehabilitation bei Multipler Sklerose in Deutschland: Ergebnisse einer Umfrage. Aktuelle Neurologie 37, 4-9.
Hellwig K, Haghikia A, Rockhoff M, Gold R (2012) Multiple sclerosis and pregnancy: experience from a nationwide database in Germany. Ther Adv Neurol Disord 5(5), 247-253. DOI: 10.1177/1756285612453192.

Hellwig K, Brune N, Haghikia A, Muller T, Schimrigk S, chwodiauer V, Gold R (2008) Reproductive counselling, treatment and course of pregnancy in 73 German MS patients. Acta Neurol Scand 118(1), 24-28. DOI: 10.1111/ j.1600-0404.2007.00978.x.

Hellwig K, Gold R (2008) Breastfeeding and multiple sclerosis in a German cohort. Mult Scler 14(5), 718. DOI: 10.1177/ 1352458507087847.

Henze T, Flachenecker P, Zettl U (2013) Bedeutung und Behandlung der Spastik bei Multipler Sklerose: Ergebnisse der MOVE-1-Studie. Nervenarzt 84(2), 214-222. DOI: 10.1007/s00115-012-3724-1.

Höer A, Schiffhorst G, Zimmermann A, Fischaleck J, Gehrmann L, Ahrens H, Carl G, Sigel KO, Osowski U, Klein M, Bless HH (2014) Multiple sclerosis in Germany: data analysis of administrative prevalence and healthcare delivery in the statutory health system. BMC Health Serv Res 14, 381. DOI: 10.1186/1472-6963-14-381.

Hurwitz BJ (2011) Analysis of current multiple sclerosis registries. Neurol 76(1 Suppl 1), S7-13. DOI: 10.1212/ WNL.0b013e31820502f6.

IGES Institut (2014) Neurologische und psychiatrische Versorgung aus sektorenübergreifender Perspektive. Studie im Auftrag von Berufsverband Deutscher Nervenärzte e.V. (BVDN), Berufsverband Deutscher Neurologen e.V. (BDN), Berufsverband Deutscher Psychiater e.V (BVDP), Kassenärztliche Bundesvereinigung (KBV) / Zentralinstitut für die kassenärztliche Versorgung in Deutschland (ZI), Deutsche Gesellschaft für Neurologie e.V (DGN). Ergebnisbericht. Berlin: IGES Institut

InEK (2015) G-DRG-Browser 2013_2014. Siegburg: Institut für das Entgeltsystem im Krankenhaus http://www.gdrg.de/ cms/Datenveroeffentlichung_gem._21_KHEntgG [Abruf am: 29. Oktober 2015].

Kasten E, Eder R, Robra B-P, Sabel BA (1997) Der Bedarf an ambulanter neuropsychologischer Behandlung. Neuropsychol 8(1), 72-85. ISSN: 1016-264X.

Kerling A, Keweloh K, Tegtbur U, Kuck M, Grams L, Horstmann $\mathrm{H}$, Windhagen A (2015) Effects of a Short Physical Exercise Intervention on Patients with Multiple Sclerosis (MS). Int J Mol Sci 16(7), 15761-15775. DOI: 10.3390/ijms160715761.

Khan F, Amatya B, Kesselring J, Galea MP (2015) Telerehabilitation for persons with multiple sclerosis. A Cochrane review. Eur J Phys Rehabil Med 51(3), 311-325. ISSN: 1973-9087.

Khan F, Turner-Stokes L, Ng L, Kilpatrick T (2007) Multidisciplinary rehabilitation for adults with multiple sclerosis. The Cochrane Database of Syst Rev (2), CD006036. DOI: 10.1002/14651858.CD006036.pub2.

Khil L, Flachenecker P, Zettl U, Elias W, Freidl M, Haas J, Pitschnau-Michel D, Schimrigk S, Rieckmann P (2009) Update on the German MS Register - Immunotherapy and drug discontinuation. 19th Meeting oft he European Neorological Society, 20.06.2009-24.06.2009. 
Kopke S, Kern S, Ziemssen T, Berghoff M, Kleiter I, Marziniak M, Paul F, Vettorazzi E, Pottgen J, Fischer K, Kasper J, Heesen C (2014) Evidence-based patient information programme in early multiple sclerosis: a randomised controlled trial. J Neurol Neurosurg Psychiatry 85(4), 411-418. DOI: 10.1136/jnnp-2013-306441.

Kopke S, Richter T, Kasper J, Muhlhauser I, Flachenecker P, Heesen C (2012) Implementation of a patient education program on multiple sclerosis relapse management. Patient Educ Couns 86(1), 91-97. DOI: 10.1016/j. pec.2011.03.013.

KV Hessen (2014) Qualitätssicherung und Genehmigungspflicht. Neuropsychologische Therapie. Frankfurt: Kassenärztliche Vereinigung Hessen http://www.kvhessen. de/fuer-unsere-mitglieder/qualitaet/qualitaetssicherungund-genehmigungspflicht/neuropsychologische-therapie/ [Abruf am: 15.09.2015].

Latimer-Cheung AE, Pilutti LA, Hicks AL, Martin Ginis KA, Fenuta AM, MacKibbon KA, Motl RW (2013) Effects of Exercise Training on Fitness, Mobility, Fatigue, and Health-Related Quality of Life Among Adults With Multiple Sclerosis: A Systematic Review to Inform Guideline Development. Arch Phys Med Rehabil 94(9), 1800-1828. DOI: 10.1016/j.apmr.2013.04.020.

Lublin FD, Reingold SC, Cohen JA, Cutter GR, Sorensen PS, Thompson AJ, Wolinsky JS, Balcer LJ, Banwell B, Barkhof $F$, Bebo B, Jr., Calabresi PA, Clanet M, Comi G, Fox RJ, Freedman MS, Goodman AD, Inglese M, Kappos L, Kieseier BC, Lincoln JA, Lubetzki C, Miller AE, Montalban X, O'Connor PW, Petkau J, Pozzilli C, Rudick RA, Sormani MP, Stuve O, Waubant E, Polman CH (2014) Defining the clinical course of multiple sclerosis: the 2013 revisions. Neurol 83(3), 278-286. DOI: 10.1212/WNL.0000000000000560.

Maurer M, Dachsel R, Domke S, Ries S, Reifschneider G, Friedrich $A$, Knorn $P$, Landefeld $H$, Niemczyk G, Schicklmaier $P$, Wernsdorfer C, Windhagen S, Albrecht H, Schwab S, TYPIC Study Investigators (2011) Health care situation of patients with relapsing-remitting multiple sclerosis receiving immunomodulatory therapy: a retrospective survey of more than 9000 German patients with MS. Eur J Neurol 18(8), 1036-1045. DOI: 10.1111/j.14681331.2010.03313.x.

Mühlig S, Rother A, Neumann-Thiele A, Scheurich A (2009) Zur Versorgungssituation im Bereich der ambulanten neuropsychologischen Therapie - eine bundesweite Totalerhebung. Z Neuropsychol 20(2), 93-107. DOI: 10.1024/ 1016-264X.20.2.93.

Mutschler E, Geisslinger G, Kroemer HK, Menzel S, Ruth P (2013) Mutschler Arzneimittelwirkungen: Lehrbuch der Pharmakologie, der klinischen Pharmakologie und Toxikologie; mit einführenden Kapiteln in die Anatomie, Physiologie und Pathophysiologie. 10. vollständig überarbeitete und erweiterte Auflage. Stuttgart: Wissenschaftliche Verlagsgesellschaft $\mathrm{mbH}$.

Nagaraj K, Taly AB, Gupta A, Prasad C, Christopher R (2013) Depression and sleep disturbances in patients with multiple sclerosis and correlation with associated fatigue.
J Neurosci Rural Pract 4(4), 387-391. DOI: 10.4103/09763147.120201.

O'Brien AR, Chiaravalloti N, Goverover Y, Deluca J (2008) Evidenced-based cognitive rehabilitation for persons with multiple sclerosis: a review of the literature. Arch Phys Med Rehabil 89(4), 761-769. DOI: 10.1016/ j.apmr.2007.10.019.

Patejdl R, Penner IK, Noack TK, ZettI UK (2015) [Fatigue in patients with multiple sclerosis--pathogenesis, clinical picture, diagnosis and treatment]. Fortschr Neurol Psychiatr 83(4), 211-220. DOI: 10.1055/s-0034-1399353.

Pearson M, Dieberg G, Smart N (2015) Exercise as a Therapy for Improvement of Walking Ability in Adults With Multiple Sclerosis: A Meta-Analysis. Arch Phys Med Rehabil 96(7), 1339-1348. DOI: 10.1016/j.apmr.2015.02.011.

Pecori C, Giannini M, Portaccio E, Ghezzi A, Hakiki B, Pasto L, Razzolini L, Sturchio A, De Giglio L, Pozzilli C, Paolicelli D, Trojano M, Marrosu MG, Patti F, Mancardi GL, Solaro C, Totaro R, Tola MR, De Luca G, Lugaresi A, Moiola L, Martinelli V, Comi G, Amato MP, MS Study Group of the Italian Neurological Society (2014) Paternal therapy with disease modifying drugs in multiple sclerosis and pregnancy outcomes: a prospective observational multicentric study. BMC Neurol 14, 114. DOI: 10.1186/1471-2377-14-114.

Penner IK, Kappos L, Rausch M, Opwis K, Radu EW (2006) Therapy-induced plasticity of cognitive functions in MS patients: insights from fMRI. J Physiol, Paris 99(4-6), 455-462. DOI: 10.1016/j.jphysparis.2006.03.008.

Pottgen J, Lau S, Penner I, Heesen C, Moritz S (2015) Managing Neuropsychological Impairment in Multiple Sclerosis: Pilot Study on a Standardized Metacognitive Intervention. Int J MS Care 17(3), 130-137. DOI: 10.7224/15372073.2014-015.

Remington G, Rodriguez Y, Logan D, Williamson C, Treadaway $\mathrm{K}$ (2013): Facilitating medication adherence in patients with multiple sclerosis. Int J MS Care 15(1), 36-45. DOI: 10.7224/1537-2073.2011-038.

Rommer PS, Buckow K, Ellenberger D, Friede T, PitschnauMichel D, Fuge J, Stuve O, Zettl UK, German Multiple Sclerosis Registry of the German National Multiple Sclerosis Society (2015) Patients characteristics influencing the longitudinal utilization of steroids in multiple sclerosis - an observational study. Eur J Clin Invest 45(6), 587-593. DOI: 10.1111/eci.12450.

Rosti-Otajarvi EM, Hamalainen PI (2014) Neuropsychological rehabilitation for multiple sclerosis. Cochrane Database Syst Rev 2, CD009131. DOI: 10.1002/14651858.CD009131. pub3.

Russel WR, Palfrey G (1969) Disseminated sclerosis: restexercise therapya progress report. Physiother 55(8), 306-310.

Sachverständigenrat zur Begutachtung der Entwicklung im Gesundheitswesen (2014) Bedarfsgerechte Versorgung - Perspektiven für ländliche Regionen und ausgewählte Leistungsbereiche. http://www.svr-gesundheit.de/file admin/user_upload/Gutachten/2014/SVR-Gutachten_2014_Langfassung.pdf [Abruf am: 30. April 2015]. 
Scalfari A, Neuhaus A, Daumer M, Muraro PA, Ebers GC (2014) Onset of secondary progressive phase and long-term evolution of multiple sclerosis. J Neurol Neurosurg Psychiatry 85(1), 67-75. DOI: 10.1136/jnnp-2012-304333.

Scalfari A, Neuhaus A, Degenhardt A, Rice GP, Muraro PA, Daumer M, Ebers GC (2010) The natural history of multiple sclerosis: a geographically based study 10: relapses and long-term disability. Brain 133(Pt 7), 1914-1929. DOI: 10.1093/brain/awq118.

Schubert I, Koster I, Kupper-Nybelen J, Ihle P (2008) [Health services research based on routine data generated by the SHI. Potential uses of health insurance fund data in health services research]. Bundesgesundheitsblatt 51(10), 1095-1105. DOI: 10.1007/s00103-0080644-0.

Schwabe U, Paffrath D (2014) Arzneiverordnungs-Report 2014. Berlin Heidelberg: Springer-Verlag. ISBN: 978-3662-43487-1.

Simonow A (2015) Multiple Sklerose: Was hilft jenseits von Medikamenten?- MS-Komplexbehandlung oder Reha? -. [Präsentation] 14. Hamburger MS -Forum. 30. Mai 2015. Hamburg-Schnelsen. http://www.dmsg-hamburg.de/ wp-content/uploads/2015/06/DMSG-HH_20153005.pdf [Abruf am: 14. Dezember 2015].

Spitzenverbände der Krankenkassen, Bundesarbeitsgemeinschaft der Heilmittelverbände e.V. (BHV), Deutscher Bundesverband der Atem- S-uSi, Lehrervereinigung Schlaffhorst-Andersen e.V. (dba), Deutscher Bundesverband der akademischen Sprachtherapeuten e.V. (dbs) (2006) Anlage 1a zu den Rahmenempfehlungen nach $\S 125$ Abs. 1 SGB V vom 1. August 2001 in der Fassung vom 1. Juni 2006: Leistungsbeschreibung Physiotherapie. https://www.gkv-spitzenverband.de/media/dokumente/ krankenversicherung_1/ambulante_leistungen/heilmittel/heilmittel_rahmenempfehlungen/125_Anlage_1a 208.pdf [Abruf am: 14. Dezember 2015].

Stangel M, Penner IK, Kallmann BA, Lukas C, Kieseier BC (2015) Towards the implementation of 'no evidence of disease activity' in multiple sclerosis treatment: the multiple sclerosis decision model. Ther Adv Neurol Disord 8(1), 3-13. DOI: $10.1177 / 1756285614560733$.

Statistisches Bundesamt (2013) Gesundheit. Grunddaten der Vorsorge- oder Rehabilitationseinrichtungen. Fachserie 12 Reihe 6.1.2. Wiesbaden: Statistisches Bundesamt.

Summers M, Fisniku L, Anderson V, Miller D, Cipolotti L, Ron M (2008) Cognitive impairment in relapsing-remitting multiple sclerosis can be predicted by imaging performed several years earlier. Mult Scler 14(2), 197-204. DOI: 10.1177/1352458507082353.

Tallner A, Mäurer M, Pfeifer K (2013) Multiple Sklerose und körperliche Aktivität: Eine historische Betrachtung. Der Nervenarzt 84(10), 1238-1244. DOI: 10.1007/s00115-0133838-0.

Tarrants M, Oleen-Burkey M, Castelli-Haley J, Lage MJ (2011) The impact of comorbid depression on adherence to therapy for multiple sclerosis. Mult Scler Int 2011, 271321. DOI: $10.1155 / 2011 / 271321$.
Twork S, Nippert I, Scherer P, Haas J, Pohlau D, Kugler J (2007) Immunomodulating drugs in multiple sclerosis: compliance, satisfaction and adverse effects evaluation in a German multiple sclerosis population. Curr Med Res Opin 23(6), 1209-1215. DOI: 10.1185/030079907X 188125.

Vogel H-P (2015) Die Behandlung der Multiplen Sklerose - der Stand heute. Arzneiverordnung in der Praxis 42(1).

Vogt A, Kappos L, Calabrese P, Stöcklin M, Gschwind L, Opwis K, Penner I (2008) Wirksamkeit eines neu entwickelten kognitiven Trainingsprogramms bei MS. Neurol Rehabil 14(2), 95-103.

Vogt A, Kappos L, Calabrese P, Stocklin M, Gschwind L, Opwis $\mathrm{K}$, Penner IK (2009) Working memory training in patients with multiple sclerosis - comparison of two different training schedules. Restor Neurol Neurosci 27(3), 225-235. DOI: 10.3233/RNN-2009-0473.

Weinshenker BG, Bass B, Rice GP, Noseworthy J, Carriere W, Baskerville J, Ebers GC (1989) The natural history of multiple sclerosis: a geographically based study. I. Clinical course and disability. Brain 112 (Pt 1), 133-146. ISSN: 0006-895.

Westerlind H, Ramanujam R, Uvehag D, Kuja-Halkola R, Boman M, Bottai M, Lichtenstein P, Hillert J (2014) Modest familial risks for multiple sclerosis: a registry-based study of the population of Sweden. Brain 137(Pt 3), 770-778. DOI: 10.1093/brain/awt356.

Wiendl H, Kieseier BC, Brandt T, (Hrsg), Hohlfeld R, (Hrsg.), Noth J, (Hrsg.), Reichmann H, (Hrsg) (2010) Multiple Sklerose. Klinik, Diagnose und Therapie. München: Kohlhammer. ISBN: 978-3170184633.

Wiendl H, Meuth SG (2015) Pharmacological Approaches to Delaying Disability Progression in Patients with Multiple Sclerosis. Drugs 75(9), 947-977. DOI: 10.1007/s40265-0150411-0.

Wilson LS, Loucks A, Gipson G, Zhong L, Bui C, Miller E, Owen M, Pelletier D, Goodin D, Waubant E, McCulloch CE (2015) Patient preferences for attributes of multiple sclerosis disease-modifying therapies: development and results of a ratings-based conjoint analysis. Int J MS Care 17(2), 74-82. DOI: 10.7224/1537-2073.2013-053.

Windt R (2014): Multiple Sklerose - Neue Therapieoptionen. In: Glaeske G \& Schicktanz C: BARMER GEK Arzneimittelreport 2014. Auswertungsergebnisse der BARMER GEK Arzneimitteldaten aus den Jahren 2012 bis 2013. BARMER GEK.

World Health Organization (2005) Internationale Klassifikation der Funktionsfähigkeit, Behinderung und Gesundheit. Köln: DIMDI.

Yermakov S, Davis M, Calnan M, Fay M, Cox-Buckley B, Sarda S, Duh MS, lyer R (2015) Impact of increasing adherence to disease-modifying therapies on healthcare resource utilization and direct medical and indirect work loss costs for patients with multiple sclerosis. Journal Med Econ 18(9), 1-10. DOI: 10.3111/13696998.2015.1044276. 